\title{
A New Model of the Gravitational Lens 0957+561 and a Limit on the Hubble Constant
}

\section{Citation}

Grogin, Norman A., and Ramesh Narayan. 1996. "A New Model of the Gravitational Lens 0957+561 and a Limit on the Hubble Constant." The Astrophysical Journal 464 (June): 92. https:// doi.org/10.1086/177302.

\section{Permanent link}

http://nrs.harvard.edu/urn-3:HUL.InstRepos:41384901

\section{Terms of Use}

This article was downloaded from Harvard University's DASH repository, and is made available under the terms and conditions applicable to Other Posted Material, as set forth at http:// nrs.harvard.edu/urn-3:HUL.InstRepos:dash.current.terms-of-use\#LAA

\section{Share Your Story}

The Harvard community has made this article openly available.

Please share how this access benefits you. Submit a story.

Accessibility 


\title{
A New Model of the Gravitational Lens 0957+561 and a Limit on the Hubble Constant
}

\author{
Norman A. Grogin and Ramesh Narayan \\ Harvard-Smithsonian Center for Astrophysics, 60 Garden Street, Cambridge, MA 02138 \\ E-mail: ngrogin, rnarayan@cfa.harvard.edu
}

\begin{abstract}
We present a simple mass model for the lensing galaxy in the gravitationally lensed quasar $0957+561$. We represent the galaxy as a softened power-law sphere (SPLS), a generalization of the singular isothermal sphere with three parameters - $\rho_{0}$, the central density, $\theta_{c}$, the angular core radius, and $\eta$, the radial index which is defined such that mass increases as $r^{\eta}$ at large radius. As in previous studies we approximate the galaxy cluster surrounding the lensing galaxy by means of a quadratic potential described by its convergence $\kappa$ and shear $\gamma$. A feature of the model is that it does not require a large central compact mass.

We fit the model to a recent high resolution VLBI map of the two images of $0957+561$. The data provide a number of independent constraints and the modelfit has six degrees of freedom, which is a significant improvement over previous models. Although the reduced $\chi^{2}$ of the best-fit model is only 4.3 , nevertheless we obtain a tight constraint on the radial index, $1.07<\eta<1.18$, at the $95 \%$ confidence level. Thus, the galaxy has mass increasing slightly more rapidly than isothermal $(\eta=1)$ out to at least $15 h^{-1} \mathrm{kpc}$. Since the light from the galaxy follows a de Vaucouleurs profile, we deduce that the mass-to-light ratio of the galaxy increases rapidly with increasing radius. We also obtain an upper limit on the core radius, namely $\theta_{c}<0^{\prime \prime}$. 11 or linear core radius $<330 h^{-1}$ pc.

We use the model to calculate the Hubble constant $H_{0}$ as a function of the time delay $\Delta \tau_{B A}$ between the two images. We obtain

$$
\begin{aligned}
H_{0} & =\left(60.5_{-2.2}^{+4.3}\right)(1-\kappa)\left(\Delta \tau_{B A} / 1.5 \mathrm{yr}\right)^{-1} \mathrm{~km} \mathrm{~s}^{-1} \mathrm{Mpc}^{-1}, \text { or } \\
& =\left(82.5_{-3.0}^{+5.9}\right)(1-\kappa)\left(\Delta \tau_{B A} / 1.1 \mathrm{yr}\right)^{-1} \mathrm{~km} \mathrm{~s}^{-1} \mathrm{Mpc}^{-1} .
\end{aligned}
$$
\end{abstract}

Once $\Delta \tau_{B A}$ is measured, this will provide an upper bound on $H_{0}$ since $\kappa$ cannot be negative. In addition, the model degeneracy due to $\kappa$ can be eliminated if the one-dimensional velocity dispersion $\sigma$ of the lensing galaxy is measured. In this case we find that

$$
\begin{aligned}
H_{0} & =\left(60.5_{-4.1}^{+6.4}\right)\left(\sigma / 322 \mathrm{~km} \mathrm{~s}^{-1}\right)^{2}\left(\Delta \tau_{B A} / 1.5 \mathrm{yr}\right)^{-1} \mathrm{~km} \mathrm{~s}^{-1} \mathrm{Mpc}^{-1}, \text { or } \\
& =\left(82.5_{-5.6}^{+8.7}\right)\left(\sigma / 322 \mathrm{~km} \mathrm{~s}^{-1}\right)^{2}\left(\Delta \tau_{B A} / 1.1 \mathrm{yr}\right)^{-1} \mathrm{~km} \mathrm{~s}^{-1} \mathrm{Mpc}^{-1}
\end{aligned}
$$


We find that these results are virtually unchanged when we investigate the effects of ellipticity in the lensing galaxy and clumpiness in the lensing cluster.

\section{Introduction}

In two seminal papers, Refsdal $(1964,1966)$ showed that there should exist a time delay between flux variations of multiple images of a lensed background source, and demonstrated that the time delay is inversely proportional to the Hubble constant $H_{0}$. Gravitational lenses thus can be used to determine $H_{0}$ independently of traditional distance-ladder techniques. The lens method of estimating $H_{0}$ requires a measurement of the time delay and a determination of the mass distribution of the lens.

A particularly promising candidate for this technique is the first gravitational lens discovered, the so-called "double quasar" 0957+561 (Walsh, Carswell, \& Weymann 1979), which consists of a pair of lensed images of a $z=1.41$ quasar separated by 6 " on the sky. The galaxy responsible for the lensing, denoted G1, was discovered by Stockton (1980). This galaxy is a bright cluster elliptical at redshift $z=0.36$, residing in a cluster of galaxies which also contributes to the lensing (Garrett, Walsh, \& Carswell 1992). Long-term monitoring of the optical (Vanderreist et al. 1992; Schild 1990) and radio (Lehár et al. 1992) light curves of the two quasar images has provided strong evidence for a time delay, though there is still some uncertainty regarding the actual value of the delay (Press, Rybicki, \& Hewitt 1992b,c; Schild \& Thomson 1993; ;elt et al. 1995). The observational uncertainties in the other observables such as the image positions, the relative image magnification, and the source and lens redshifts are small, leaving the lens mass distribution as the chief remaining obstacle to estimating $H_{0}$ using this system (Borgeest \& Refsdal 1984). The mass distribution of the lens is difficult to measure directly, and has to be constrained using the observations of the lensed images.

It is customary to postulate a simple functional form for the lens mass profile, and to adjust the parameters of the model so as to obtain the best fit to the observables, namely the quasar image positions and relative image magnifications. Such calculations have been done by various authors in the past (Young et al. 1980; Borgeest \& Refsdal 1984; Greenfield, Roberts, \& Burke 1985; Kochanek 1991; Falco, Gorenstein, \& Shapiro 1991, hereafter FGS; Bernstein, Tyson, \& Kochanek 1993, hereafter BTK) using the data available at the time. We present here a new model of $0957+561$ which improves on previous work in two respects.

First, we employ a new parameterization of the lens mass, namely the softened powerlaw sphere (SPLS) model, which allows us to explore a wider range of radial mass profiles 
than in earlier studies. Our model space includes the standard isothermal sphere model (e.g. Turner, Ostriker, \& Gott 1984) as a particular case, and one of our aims is to use the data to determine the degree to which the galaxy deviates from an isothermal radial profile. We also include a core radius for the galaxy and represent the surrounding cluster by means of a quadratic potential as proposed by FGS.

Second, we make use of a larger set of data constraints than in any previous study. FGS for their modeling used high-resolution VLBI maps of 0957+561 Gorenstein et al. 1988a) which resolved features of an inner radio jet extending $\sim 50$ milliarcseconds from the quasar core of each image. This permitted them to derive a $4 \times 4$ relative magnification matrix between the two quasar images which they used to constrain their model. Recent $\lambda 18 \mathrm{~cm}$ VLBI mapping of 0957+561A,B by Garrett et al. (1994, hereafter G94) revealed even greater detail in the fine structure of the quasar images. The $\mathrm{A}$ and $\mathrm{B}$ inner jet regions are now resolved into five centers of emission with sub-milliarcsecond accuracy in the positions. In addition, G94 report a gradient in the relative magnification tensor between the quasar core and the end of the inner jet. This provides two additional data constraints which we include in our model-fitting. As a result of the additional data, we have a better-constrained lens model, with six degrees of freedom in the data fits, as against the previous studies by FGS and Kochanek (1991) which had only one degree of freedom.

In $\S 2$ of the paper we briefly introduce the notation and approximations employed in this study, as well as the basic lens equations that allow us to test mass models against constraints from observation. In $\S 3$ we review the observations of $0957+561$, paying particular attention to the high-resolution VLBI mapping by G94 which provides the majority of our model constraints. We introduce the SPLS lens model in $\S 4$ and derive its lensing properties. We then employ the lensing equations of $\S 2$ to show explicitly the dependence of model-predicted observables, including the time delay, upon our various model parameters. For comparison, we do the same also for the FGS lens model. In $\S 5$ we describe the results of our model-fitting, both with our SPLS model and with the FGS model, and including the effects of ellipticity in G1 and clumpiness in the cluster. We derive confidence limits on the lens parameters and obtain bounds on $H_{0}$. In $\S 6$ we address one of the chief difficulties in obtaining tight $H_{0}$ bounds from $0957+561$, namely the uncertainty regarding the amount of lensing contributed by the cluster. We describe how this uncertainty can be removed by measuring the velocity dispersion of the galaxy, as shown by FGS, or by measuring the shape and velocity dispersion of the cluster. We summarize the paper in $\S 7$ and discuss prospects for further improvements in the method, both with $0957+561$ and with other lens systems. 


\section{Lensing Geometry and Notation}

We employ a Cartesian coordinate system on the sky with the origin at the center of mass of the lensing galaxy G1, the $x$-axis taken positive to the east, and the $y$-axis positive to the north. We define position angles to be zero toward the north and increasing eastward. We employ the standard angular diameter distance appropriate to a Friedmann universe. For an observer at redshift $z_{i}$ and a source at redshift $z_{j}$, the angular diameter distance is given by

$$
D\left(z_{i}, z_{j}\right)=\frac{\xi_{j}}{\theta_{i}}=\frac{2 c}{H_{0}} \frac{\left(1-\Omega_{0}-G_{i} G_{j}\right)\left(G_{i}-G_{j}\right)}{\Omega_{0}^{2}\left(1+z_{i}\right)\left(1+z_{j}\right)^{2}}, \quad G_{i, j} \equiv\left(1+\Omega_{0} z_{i, j}\right)^{1 / 2},
$$

where $H_{0}=100 h \mathrm{~km} \mathrm{~s}^{-1} \mathrm{Mpc}^{-1}$ is the Hubble constant. A proper length $\xi$ at $z_{j}$ subtends an angle $\theta=\xi / D\left(z_{i}, z_{j}\right)$ at $z_{i}$. It is customary to use the abbreviations $D_{d} \equiv D\left(0, z_{d}\right)$, $D_{s} \equiv D\left(0, z_{s}\right)$, and $D_{d s} \equiv D\left(z_{d}, z_{s}\right)$ when referring to the angular diameter distances from observer to deflector, observer to source, and deflector to source, respectively. We also introduce the effective lens distance

$$
D \equiv D_{d} D_{s} / D_{d s}
$$

which appears in the lensing equations below.

For simplicity, we assume $\Omega_{0}=1$ in what follows. The sensitivity of the results to $\Omega_{0}$ is rather small (FGS). For the $0957+561$ lens, $D$ increases approximately linearly with decreasing $\Omega_{0}$, to a value $\sim 8 \%$ larger for $\Omega_{0}=0$ than the fiducial value for $\Omega_{0}=1$. A nonzero cosmological constant $\Lambda$ can have a more important effect on the results (e.g. Turner 1990), but we do not explore the dependence in this paper.

Using standard notation (e.g. Blandford \& Narayan 1992; Schneider, Ehlers, \& Falco 1992), we represent angular positions at the distance of the background source (the "source plane") with the vector $\boldsymbol{\beta}$ and angular positions at the distance of the deflector (the "image plane") with $\boldsymbol{\theta}$. The lens mass deflects light rays at the image plane through an angle which we represent with $\hat{\boldsymbol{\alpha}}$. These quantities are then related via the lens equation,

$$
\boldsymbol{\beta}=\boldsymbol{\theta}-\boldsymbol{\alpha}(\boldsymbol{\theta}),
$$

where the reduced deflection angle $\boldsymbol{\alpha}(\boldsymbol{\theta})$ is related to the true deflection angle $\hat{\boldsymbol{\alpha}}(\boldsymbol{\theta})$ at the image plane by

$$
\boldsymbol{\alpha}=\left(\frac{D_{d s}}{D_{s}}\right) \hat{\boldsymbol{\alpha}}=\left(\frac{D_{d}}{D}\right) \hat{\boldsymbol{\alpha}}
$$

The ray deflection function $\boldsymbol{\alpha}(\boldsymbol{\theta})$ may in general allow multiple solutions $\boldsymbol{\theta}_{i}$ to the lens equation 2-3] for a given $\boldsymbol{\beta}$. If a source happens to lie at such a $\boldsymbol{\beta}$, we observe multiple images of the source at positions $\boldsymbol{\theta}_{i}$, a gravitational "mirage". 
The lens deflection $\hat{\boldsymbol{\alpha}}(\boldsymbol{\theta})$ is sensitive only to the surface mass density of the deflector. With $\boldsymbol{\xi} \equiv D_{d} \boldsymbol{\theta}$ representing linear position in the image plane, we may express the lens deflection angle as the two-dimensional gradient of a potential $\psi(\boldsymbol{\xi})$,

$$
\hat{\boldsymbol{\alpha}}(\boldsymbol{\xi})=\nabla_{\boldsymbol{\xi}} \psi / c^{2} .
$$

The potential $\psi$ is related to the lens surface mass density $\Sigma$ according to $\nabla_{\xi}^{2} \psi(\boldsymbol{\xi})=8 \pi G \Sigma(\boldsymbol{\xi})$. Equivalently, we may express the deflection in terms of the Green's function of the Poisson operator,

$$
\hat{\boldsymbol{\alpha}}(\boldsymbol{\xi})=\iint d^{2} \boldsymbol{\xi}^{\prime}\left(\frac{4 G \Sigma\left(\boldsymbol{\xi}^{\prime}\right)}{c^{2}}\right) \frac{\boldsymbol{\xi}-\boldsymbol{\xi}^{\prime}}{\left|\boldsymbol{\xi}-\boldsymbol{\xi}^{\prime}\right|^{2}} .
$$

For a radially symmetric surface mass density profile $\Sigma(\xi)$, the above equation simplifies to

$$
\hat{\boldsymbol{\alpha}}(\boldsymbol{\xi})=\left(\frac{4 G M(\xi)}{c^{2} \xi^{2}}\right) \boldsymbol{\xi}
$$

where $M(\xi)$ is the projected mass of the deflector within cylindrical radius $\xi$.

Gravitational lensing not only causes images of a background source to appear at different positions, but the images are also magnified or demagnified. In general the magnification is anisotropic, and is described by a symmetric (Bourassa \& Kantowski 1975) $2 \times 2$ magnification tensor $\left[\mathcal{M}^{i}\right]$ which is given by

$$
\left[\mathcal{M}^{i}\right]=\left[\frac{\partial \boldsymbol{\theta}}{\partial \boldsymbol{\beta}}\right]_{\boldsymbol{\theta}_{i}}=\left[\mathcal{I}_{2}-\left.\frac{\partial \boldsymbol{\alpha}}{\partial \boldsymbol{\theta}}\right|_{\boldsymbol{\theta}_{i}}\right]^{-1}
$$

Here $\mathcal{I}_{2}$ is the $2 \times 2$ identity matrix. We arrive at the leftmost expression via differentiation of the lensing equation [2-3]. Because we cannot view the unlensed source, we cannot determine $\left[\mathcal{M}^{i}\right]$ from observations of the system. If however the background source is multiply imaged and the images are resolved, then we can directly measure the relative magnification tensor from one image to another, $\left[\mathcal{M}^{i j}\right]=\left(\partial \boldsymbol{\theta}_{i} / \partial \boldsymbol{\theta}_{j}\right)$. The relative magnification is related to the lens deflection function $\boldsymbol{\alpha}$ via equation 2-8 above:

$$
\left[\mathcal{M}^{i j}\right] \equiv\left[\mathcal{M}^{i}\right]\left[\mathcal{M}^{j}\right]^{-1}=\left[\mathcal{I}_{2}-\left.\frac{\partial \boldsymbol{\alpha}}{\partial \boldsymbol{\theta}}\right|_{\boldsymbol{\theta}_{i}}\right]^{-1}\left[\mathcal{I}_{2}-\left.\frac{\partial \boldsymbol{\alpha}}{\partial \boldsymbol{\theta}}\right|_{\boldsymbol{\theta}_{j}}\right]
$$

Although the magnification tensor is symmetric, the relative magnification tensor is not and thus will have four independent components. It is customary to refer to the relative magnification tensor in terms of its eigenvalues $M_{1,2}$ and corresponding eigenvector position angles $\phi_{1,2}$ (e.g. FGS). With unresolved images, it is not possible to measure the full relative magnification tensor. However, the flux ratio of the two images gives the magnitude of the determinant of $\left[\mathcal{M}^{i j}\right]$. 


\section{Observational Constraints}

\subsection{Image Positions and Magnifications}

The highest-resolution images of $0957+561$ have been obtained by means of radio VLBI. Shortly after the discovery of the source, VLBI observations (Porcas et al. 1981) revealed similar core-jet structure in the radio components A and B, reinforcing the lensing hypothesis for the system. Gorenstein et al. (1988a) obtained improved $\lambda 13 \mathrm{~cm}$ VLBI maps and resolved the $\mathrm{A}$ and $\mathrm{B}$ jet images into three Gaussian components each. They were thereby able to construct the magnification tensor $\left[M_{B A}\right]$ relating the $\mathrm{A}$ and $\mathrm{B}$ images. Implicit in their calculation of $\left[M_{B A}\right]$ was the assumption that the tensor remains the same both in the QSO core and in the jet region. G94 have recently obtained even more accurate VLBI maps of $0957+561$ at $\lambda 18 \mathrm{~cm}$ and have been able to measure variations of $\left[M_{B A}\right]$ along the jet.

G94 fit the A and B VLBI images of $0957+561$ with six Gaussian components each, denoted as $A_{1 \ldots 6}$ and $B_{1 \ldots 6} . A_{1}$ and $B_{1}$ correspond to the respective core components while $A_{2 \ldots 6}$ and $B_{2 \ldots 6}$ are successive blobs in the jet. The relative positions of the brightest jet components, $A_{5}$ and $B_{5}$, with respect to the core components, $A_{1}$ and $B_{1}$, are measured to within 0.1 mas (Table 1). In our lens modeling, we use these two image offsets as constraints. Because G94 do not give the separation between images $\mathrm{A}$ and $\mathrm{B}$, we take as $\left(A_{1}-B_{1}\right)$ the

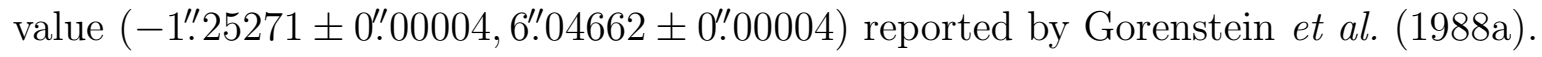

The improved resolution of the $\lambda 18 \mathrm{~cm}$ VLBI map allowed G94 to measure the change in the relative magnification tensor along the axis of the jet. This gradient is effectively measured between components 1 and 5 in the two images. Because of their limited signal to noise, G94 were forced to set two of the magnification tensor gradient components to zero, and they evaluated the gradients only of the other two components. Thus, G94 provide a total of six constraints on the magnification tensor, viz. four matrix elements corresponding to the transformation from $A_{5}$ to $B_{5}$, plus gradients of the two matrix eigenvalues along the long axis of the jet. The six constraints are summarized in Table 2 .

\subsection{Third Image Flux}

Although gravitational lensing theory predicts a third image of $0957+561$ near the center of the lensing galaxy, no such image has been seen down to a $5 \sigma$ limit of $0.6 \mathrm{mJy}$, which is $1 / 30$ of the flux density of image B (Gorenstein et al. 1984). This flux limit provides an additional constraint on the lens model. Because there is some ambiguity in the non-detection of the third image flux (Gorenstein et al. 1983; Gorenstein et al. 1984; Bonometti 1985), we have 
chosen not to treat this constraint in the standard fashion. We instead adopt a weighting function that assigns no penalty to a lens model if it predicts a third image flux below the $5 \sigma$ detection limit, but where the penalty increases steeply if the predicted flux is greater than this limit. Thus, if $C / B$ is the flux ratio between the third image and the $\mathrm{B}$ image according to a lens model, we take its contribution to the $\chi^{2}$ to be

$$
\chi_{\mathrm{C} / \mathrm{B}}^{2}=\left\{\begin{array}{cl}
0 & : \quad C / B<1 / 30 \\
\frac{(C / B-1 / 30)^{2}}{(1 / 150)^{2}} & : \quad C / B>1 / 30
\end{array}\right.
$$

This unorthodox penalty assignment is a compromise between what we view as two unrealistic extremes. On the one hand, treating the $1 / 30$ flux ratio upper limit as a true $5 \sigma$ contribution to the overall $\chi^{2}$ excessively penalizes models for which the third image flux is say only at the 2 or $3 \sigma$ level and thus would likely have been dismissed as noise in the large area scanned for the third image. On the other hand, treating the $1 / 30$ flux ratio as a $1 \sigma$ penalty allows the model too much freedom to produce unrealistically bright third images. Although the particular $\chi^{2}$ assignment given above results in non-Gaussian uncertainties in the model parameter values, we feel that this penalty function is the fairest representation of the unclear observational status of the third image. We emphasize this point because different choices of the third image penalty lead to significantly different model limits on the G1 core radius. However, there is very little effect on our results for the Hubble constant.

\subsection{Position of the G1 Center of Mass}

Although the angular separation of the A and B images in $0957+561$ is known extremely accurately, the positions of these images with respect to the center of mass of G1 are not so well constrained (see Table 3). Stockton (1980) reported an optical center of brightness for G1 with a 30 milliarcsecond (mas) uncertainty. VLA observations of the region (Roberts $e$ t al. 1985) revealed a point-like source $\mathrm{G}$ with 1 mas uncertainty in the position. However, VLBI mapping of the same region (Gorenstein et al. 1988a) found no source coincident with the VLA detection, but did detect a weak (0.6 mJy) point source $\mathrm{G}^{\prime}$ some 30 mas away, again with 1 mas uncertainty. Both the VLA and VLBI sources are consistent with the optical center, but they are inconsistent with each other by many standard deviations.

In view of the discrepancy between the VLA and VLBI detections, we take the optical center of brightness of G1 and its error bars as the reference for the model fitting. Note that this only affects two coordinates, namely the two components of $\left(B_{1}-\mathrm{G} 1\right)$, since all other image positions are measured as offsets with respect to either $B_{1}$ or $A_{1}$. Our treatment of the galaxy center differs from that adopted by EGS, who selected the VLBI point source $\mathrm{G}^{\prime}$ 
as their center of mass position with 1 mas uncertainty.

\section{Lens Mass Models}

Most of our calculations are based on a five-parameter model of the $0957+561$ lens system where we represent the cluster as a simple astigmatic deflector and the galaxy as a power-law deflector with a core radius. We also test the particular five-parameter model used by FGS, who have the same form for the cluster contribution, but represent the galaxy as a King potential with an additional compact nucleus modeled as a point mass. We shall describe the lensing properties of these two models in detail below.

\subsection{The Softened Power-Law Sphere}

Our primary mass model for the lensing galaxy is an extension of the power-law mass distribution, $M(r) \propto r^{\eta}$, used previously in the modeling of lens systems (Wambsganss \& Paczynski 1994). We describe the galaxy by the following spherically symmetric volume density profile,

$$
\rho(r)=\rho_{0}\left(1+\frac{r^{2}}{r_{c}^{2}}\right)^{(\eta-3) / 2}
$$

where $r_{c}=\theta_{c} D_{d}$ is the core radius. For $r \gg r_{c}$, the mass of the galaxy varies as

$$
\frac{d M}{d r}=4 \pi \rho r^{2} \approx 4 \pi \rho_{0} r^{2}\left(\frac{r}{r_{c}}\right)^{(\eta-3)} \propto r^{\eta-1}
$$

which corresponds to $M\left(r \gg r_{c}\right) \propto r^{\eta}$. The parameter $\eta$ is restricted to lie between $\eta=0$, which corresponds to a modified Hubble profile (see below), and $\eta=2$, which corresponds to a constant surface mass density sheet. This family of softened power-law sphere (SPLS) models includes the singular isothermal sphere, which corresponds to $r_{c}=0, \eta=1$.

Although the SPLS density profile does not yield an analytic potential $\psi(r)$ or included mass $M(r)$, the deflection angle does have an analytic form. To show this, we first obtain the surface mass density profile implied by equation [4-1]:

$$
\Sigma(\xi)=2 \int_{\xi}^{\infty} \frac{\rho(r) r d r}{\sqrt{r^{2}-\xi^{2}}}=\Sigma_{0}\left(1+\frac{\xi^{2}}{r_{c}^{2}}\right)^{(\eta-2) / 2}, \quad \Sigma_{0} \equiv \rho_{0} r_{c} B\left(\frac{1}{2}, 1-\frac{\eta}{2}\right),
$$

where $B$ is the standard Euler beta function. This surface density is a generalization of the modified Hubble profile (cf. Binney \& Tremaine 1987), which is the specific case $\eta=0$. We 
then find that equation 44-3] yields an analytic projected mass $M(\xi)$ of the form

$$
M(\xi)=2 \pi \int_{0}^{\xi} \Sigma\left(\xi^{\prime}\right) \xi^{\prime} d \xi^{\prime}=M_{0}\left[\left(1+\frac{\xi^{2}}{r_{c}^{2}}\right)^{\eta / 2}-1\right], \quad M_{0} \equiv\left(\frac{2 \pi}{\eta}\right) \Sigma_{0} r_{c}^{2}
$$

Combining the deflection formula (eq. 2-7]) for a spherically symmetric deflector with equation 4-4, we then obtain the deflection function $\boldsymbol{\alpha}(\boldsymbol{\theta})$ :

$$
\boldsymbol{\alpha}(\boldsymbol{\theta})=\left(\frac{\alpha_{0}^{2}}{\theta^{2}}\right)\left[\left(1+\frac{\theta^{2}}{\theta_{c}^{2}}\right)^{\eta / 2}-1\right] \boldsymbol{\theta}, \quad \alpha_{0} \equiv\left(\frac{4 G M_{0}}{c^{2} D}\right)^{1 / 2}
$$

For our model-fitting we adopt an equivalent but more convenient expression for the deflection function (eq. [4-5]) which remains well-behaved even when $r_{c} \rightarrow 0$ and the central density $\rho_{0}$ diverges:

$$
\boldsymbol{\alpha}(\boldsymbol{\theta})=\left(\frac{\alpha_{\mathrm{E}}^{2}}{\theta^{2}}\right)\left[\frac{\left(\theta^{2}+\theta_{c}^{2}\right)^{\eta / 2}-\theta_{c}^{\eta}}{\alpha_{\mathrm{E}}^{\eta}}\right] \boldsymbol{\theta}, \quad \alpha_{\mathrm{E}} \equiv \alpha_{0}^{2 /(2-\eta)} \theta_{c}^{-\eta /(2-\eta)} .
$$

The parameter $\alpha_{\mathrm{E}}$ represents an approximate Einstein radius of the galaxy, insofar as $\alpha\left(\alpha_{\mathrm{E}}\right)=\alpha_{\mathrm{E}}$ for $\theta_{c}=0$, and $\alpha\left(\alpha_{\mathrm{E}}\right) \approx \alpha_{\mathrm{E}}$ for $\theta_{c} \neq 0$.

Note that the basic SPLS galaxy model described here has three adjustable parameters: $\alpha_{E}, \theta_{c}, \eta$. For the particular calculations described in $\$ 5.3$ we add a point mass at the center of the galaxy to investigate if the additional freedom would allow us to obtain a better fit of the observations. In these cases, the mass model has four parameters.

\subsection{The FGS Galaxy Deflector}

In this model the lensing galaxy consists of a smooth, circularly symmetric, King-type surface density profile parameterized by its angular core radius $\theta_{c}$ and velocity dispersion $\sigma_{v}$. The deflection function employed by FGS for this profile is an analytic approximation introduced by Young et al. (1981):

$$
\begin{aligned}
\hat{\boldsymbol{\alpha}}(\boldsymbol{\theta})[\text { radians }] & =\left(\frac{\sigma_{v}^{2}}{c^{2}}\right)\left(\frac{\boldsymbol{\theta}}{\theta}\right) \alpha_{*}(\theta), \\
\alpha_{*}(\theta) & =53.2468 f\left(1.155 \frac{\theta}{\theta_{c}}\right)-44.0415 f\left(0.579 \frac{\theta}{\theta_{c}}\right), \\
f(x) & =\frac{\left(1+x^{2}\right)^{1 / 2}-1}{x} .
\end{aligned}
$$


This formula reproduces the deflection of a King profile with cutoff radius $\theta_{t} \sim 600 \theta_{c}$ and is accurate to $\sim 1 \%$ within $10 \theta_{c}$ of the center. Because $0957+561 \mathrm{~A}$ lies $\sim 6^{\prime \prime}$ from the center of the galaxy G1 (we will hereafter refer to the point at the center as G1), model-fitting with this approximation is reliable only for $\theta_{c} \gtrsim 0^{\prime \prime} 6$.

FGS found that a pure King-type galaxy has too "soft" a potential to reproduce the observed magnification ratio between the A and B quasar images satisfactorily. Furthermore, the potential does not sufficiently demagnify the third image near the galaxy center. For both reasons FGS added to their mass model a compact nucleus at G1. This component is modeled as a point-mass in the lensing equations, with a deflection law given by

$$
\boldsymbol{\alpha}(\boldsymbol{\theta})=\left(\frac{\alpha_{\mathrm{bh}}^{2}}{\theta^{2}}\right) \boldsymbol{\theta}
$$

where $\alpha_{\mathrm{bh}}$ is the Einstein radius of the compact nucleus. The Einstein radius is related to the mass $M_{\mathrm{bh}}$ of the compact nucleus via

$$
\alpha_{\mathrm{bh}}=\left(\frac{4 G M_{\mathrm{bh}}}{c^{2} D}\right)^{1 / 2}=0^{\prime \prime} 94\left(\frac{M_{\mathrm{bh}}}{10^{11} M_{\odot}}\right)^{1 / 2}\left(\frac{D}{1 \mathrm{Gpc}}\right)^{-1 / 2} .
$$

Although treated as a point mass, the compact nucleus postulated for the $0957+561$ lens need only have a radius small in comparison with the $\approx 1^{\prime \prime}$ separation between image $\mathrm{B}$ and G1.

As in the case of the SPLS model described earlier, the FGS model again has three adjustable parameters: $\sigma_{v}, \theta_{c}, M_{\mathrm{bh}}$.

\subsection{External Sources of Deflection}

The lensing galaxy in $0957+561$ is a member of a cluster of galaxies, and because of the large angular separation of the A and B images it would appear that part of the deflection in this lens is produced by the cluster. Following FGS, we model the cluster deflection by means of a convergence $\kappa$ and shear $\gamma$ with position angle $\phi$. Such a model is reasonable so long as the projected mass density of the cluster is relatively constant over the angular scale of the image separation. We consider the effect of deviations from this model in $\$ 5.5$.

The convergence $\kappa$ is the ratio of the local mass surface density of the cluster to the critical density, $\Sigma_{\mathrm{cr}}=c^{2} D_{s} / 4 \pi G D_{d} D_{d s}$ (Blandford \& Narayan 1992). The angular deflection due to a constant convergence takes the simple form $\boldsymbol{\alpha}(\boldsymbol{\theta})=\kappa \boldsymbol{\theta}$. Since this corresponds to isotropic focusing, there is a degeneracy in lens models which was noted by Falco et al. (1985). Given any model of the lensing galaxy which fits the observations, it is possible to find a new 
model where a constant convergence $\kappa$ of arbitrary magnitude is included and the mass in the galaxy is at the same time scaled down by a factor $(1-\kappa)$. For the particular galaxy models described earlier, the parameters which are modified are: $\alpha_{\mathrm{bh}}^{2} \mapsto \alpha_{\mathrm{bh}}^{2}(1-\kappa), \alpha_{\mathrm{E}}^{2} \mapsto \alpha_{\mathrm{E}}^{2}(1-\kappa)$, $\sigma_{v}^{2} \mapsto \sigma_{v}^{2}(1-\kappa)$. All observables are invariant under this transformation, except the time delay which scales as $(1-\kappa)$. The effect on the time delay translates to a change in the derived Hubble constant: $H_{0} \mapsto H_{0}(1-\kappa)$. Since the surface mass density of the cluster has to be a positive quantity, we have the constraint $\kappa \geq 0$.

Because of the existence of the simple tranformation described above, it is not necessary to include $\kappa$ explicitly in the model. The shear, however, must be included. It transforms with variable $\kappa$ as follows: $\gamma \mapsto \gamma^{\prime}=\gamma /(1-\kappa)$. Therefore, in effect, it is the scaled shear $\gamma^{\prime}$ which we fit in our models. The deflection law due to shear takes the form

$$
\boldsymbol{\alpha}(\boldsymbol{\theta})=\gamma^{\prime}[\mathbf{T}(\phi)] \boldsymbol{\theta}, \quad \text { where } \quad[\mathbf{T}(\phi)] \equiv\left(\begin{array}{cc}
\cos 2 \phi & \sin 2 \phi \\
\sin 2 \phi & -\cos 2 \phi
\end{array}\right) .
$$

Purely from measurements of the lensed images it is not possible to break the model degeneracy due to a variable $\kappa$. However, direct measurements of the mass either in the galaxy or the cluster do offer the possibility of breaking the degeneracy. We discuss this in $\S 6$.

\subsection{Deriving $H_{0}$ from a Lens Model}

To determine a value for $H_{0}$ from $0957+561$, it is necessary to compute the difference in light propagation time from the source to the observer along the two image paths. This quantity depends on the image positions and relevant redshifts as well as the mass distribution of the lens. In the following discussion, we assume $\kappa=0$ for simplicity, and include the relevant factor of $(1-\kappa)$ only at the end.

To obtain the relative time delay we first determine the delay of the path corresponding to a given image location $\boldsymbol{\theta}_{i}$ relative to the "unlensed" light path to the observer from the source at location $\boldsymbol{\beta}$. This takes the form

$$
\tau_{i}=\frac{\left(1+z_{d}\right) D}{2 c}\left|\boldsymbol{\theta}_{i}-\boldsymbol{\beta}\right|^{2}-\frac{\left(1+z_{d}\right)}{c^{3}} \psi\left(\boldsymbol{\theta}_{i}\right),
$$

where the first term is the geometric time delay and the second is the gravitational time delay due to the "Shapiro effect" (Shapiro 1964). The factor $\left(1+z_{d}\right)$ accounts for the expansion of the universe since the deflection of the rays at redshift $z_{d}$. The relative time delay between

the two images is given by $\Delta \tau_{i j}=\tau_{i}-\tau_{j}$. Given an SPLS mass model and a fitted source 
position $\boldsymbol{\beta}$, we then have the following relation for $\Delta \tau_{i j}$ :

$$
\begin{aligned}
\frac{c \Delta \tau_{i j}}{\left(1+z_{d}\right) D}= & \frac{1}{2}\left[|\boldsymbol{\theta}-\boldsymbol{\beta}|^{2}-\gamma^{\prime}\left(\theta_{x}^{2} \cos 2 \phi-\theta_{y}^{2} \cos 2 \phi+2 \theta_{x} \theta_{y} \sin 2 \phi\right)\right]_{\boldsymbol{\theta}_{j}}^{\boldsymbol{\theta}_{i}} \\
& -\alpha_{\mathrm{E}}^{2-\eta} \int_{\boldsymbol{\theta}_{j}}^{\boldsymbol{\theta}_{i}} \frac{\left(\theta^{2}+\theta_{c}^{2}\right)^{\eta / 2}-\theta_{c}^{\eta}}{\theta} d \theta-\alpha_{\mathrm{bh}}^{2} \ln \frac{\left|\boldsymbol{\theta}_{i}\right|}{\left|\boldsymbol{\theta}_{j}\right|}
\end{aligned}
$$

where $\theta_{x}$ and $\theta_{y}$ are the $x$ - and $y$-components of angular position vector $\boldsymbol{\theta}$ in our coordinate system. We have cast the time delay equation in the above form to show the separate dependencies on model parameters (on the right) and on the effective lens distance $D$ (on the left). All other lensing variables appearing in this equation $\left(z_{d}, \boldsymbol{\theta}_{i}, \boldsymbol{\theta}_{j}\right)$ are observables. As the King-type galaxy approximation used by FGS has an analytic potential, the time delay equation for the FGS model has the following closed form:

$$
\begin{aligned}
\frac{c \Delta \tau_{i j}}{\left(1+z_{d}\right) D}= & \frac{1}{2}\left[|\boldsymbol{\theta}-\boldsymbol{\beta}|^{2}-\gamma^{\prime}\left(\theta_{x}^{2} \cos 2 \phi-\theta_{y}^{2} \cos 2 \phi+2 \theta_{x} \theta_{y} \sin 2 \phi\right)\right]_{\boldsymbol{\theta}_{j}}^{\boldsymbol{\theta}_{i}} \\
& -\theta_{c}\left(\frac{\sigma_{v}}{c}\right)^{2}\left[27.6636 g\left(1.155 \frac{|\theta|}{\theta_{c}}\right)-45.6437 g\left(0.579 \frac{|\theta|}{\theta_{c}}\right)\right]_{\boldsymbol{\theta}_{j}}^{\boldsymbol{\theta}_{i}} \\
& -\alpha_{\mathrm{bh}}^{2} \ln \frac{\left|\boldsymbol{\theta}_{i}\right|}{\left|\boldsymbol{\theta}_{j}\right|}, \\
g(x)= & \sqrt{1+x^{2}}-\ln \left(1+\sqrt{1+x^{2}}\right) .
\end{aligned}
$$

The dependence of the predicted $\Delta \tau_{i j}$ on $H_{0}$ is embedded in $D$, as can be seen from equation [2-1]. Thus, once the model parameters are determined with sufficient precision via model-fitting, we obtain an estimate of the quantity $H_{0} \Delta \tau_{i j}$. A measurement of $\Delta \tau_{i j}$ then leads to an estimate of the Hubble constant. Actually, because of the $\kappa$ degeneracy $(\$ 4.3)$, the formula for $\Delta \tau_{i j}$ given above should be multiplied by the undetermined factor $(1-\kappa)$. Therefore, technically we can only determine the quantity $H_{0} \Delta \tau_{i j} /(1-\kappa)$ from the model.

It is convenient to define a dimensionless number $h_{1.5}$, which allows for all of these factors:

$$
H_{0}=\left(100 h_{1.5} \mathrm{~km} \mathrm{~s}^{-1} \mathrm{Mpc}^{-1}\right)(1-\kappa)\left(\frac{1.5 \mathrm{yr}}{\Delta \tau_{i j}}\right) .
$$

Each lens model gives a unique value of $h_{1.5}$. The value of $H_{0}$ derived from this, however, depends on the measured time delay $\Delta \tau_{i j}$ and on the unknown magnitude of $\kappa$. Since we know that $\kappa \geq 0$, we see that the lens method can provide an upper bound for the Hubble constant even when there is no independent determination of $\kappa$.

Although the discussion here has focused on $0957+561$, the same ideas are applicable to any lensing system in which (i) we know the source and deflector redshifts (in order to 
express $D$ in terms of $H_{0}$ ), (ii) we are able to measure a time delay, and (iii) we obtain a well-constrained mass model for the lens. If we have a lens system with $C$ quasar images, we can in principle measure $(C-1)$ independent time delays, all of which should be consistent with a single value for the Hubble constant.

\section{Results}

The various observations detailed in $\S 3$ furnish a total of fifteen constraints on the mass model. Both the SPLS model and the FGS model fit these constraints with nine parameters - four source emission coordinates, two each for blob 1 and blob 5, and five variables specifying the lens mass distribution. We also study a variant of the SPLS model that includes a compact nucleus, for a total of ten parameters. The third image flux constraint discussed in $\$ 3.2$ only comes into play for models without a G1 compact nucleus. The predicted flux of the third image is zero when there is any substantial mass in the nucleus. Thus the third image flux upper limit does not influence the FGS model or the SPLS model with compact nucleus. Accordingly, we have $(15-9)=6$ degrees of freedom for the basic SPLS model, $(14-9)=5$ d.f. for the FGS model, and $(14-10)=4$ d.f. for the SPLS with compact nucleus. We discuss the results from the SPLS model in $\$ 5.1$ and those from the FGS model in $\$ 5.2$. In $\$ 5.3$, we discuss the results of adding a compact nucleus to the SPLS model. We also investigate the degree to which our SPLS model results vary if we add perturbations due to the ellipticity of G1 (\$5.4) and the influence of nearby cluster galaxies $(\S 5.5)$.

\subsection{Fitting to a Softened Power-Law Sphere}

\subsubsection{Goodness of Fit}

We employed the AMOEBA non-linear minimization algorithm (Press et al. 1992a), based on the downhill simplex method, to optimize the lens model by minimizing the $\chi^{2}$ of the fit. Our best-fit model gives $\chi^{2}=26.0$ for a chi-square per degree of freedom of $\bar{\chi}^{2}=26.0 / 6=4.3$. In Table 4 we compare the model predictions of the various observables with the measured data. In general, it is seen that the model agrees well with the data; most deviations are under $1 \sigma$ and the largest discrepancies are under $2 \sigma$. However, despite this good agreement, the formal $\bar{\chi}^{2}$ value is quite large. This is primarily the result of the very large correlations which G94 quote between the errors on the various observables (Table 1).

For instance, if we ignore the correlations and define $\chi^{2}$ to be the straight sum of the squares 
of the values in the last column of Table 4 , then our fitted parameter set gives $\chi^{2}=11.2$, corresponding to $\bar{\chi}^{2}=1.87$. Throughout this paper we always include the correlations when calculating $\chi^{2}$. G94 noted that the value of $\dot{M}_{2}$ predicted by the model of EGS is discrepant with the value which they measured. Our best-fit SPLS model has similar difficulty — the predicted $\dot{M}_{2}$ is almost two standard errors below G94's value.

An interesting feature of Table 1 is that almost all of our model $\chi^{2}$ from fitting the image positions arises from the $\left(G 1-B_{1}\right)$ constraint. As noted in 33.3 , we have adopted the larger (30 mas) error bars of the observed optical center of brightness (Stockton 1980). We find that the model takes advantage of this freedom in the center of mass position to better satisfy the other, much tighter constraints on QSO image separations from VLBI. Our best model (without compact nucleus) chooses a G1 center of mass some 62 mas from the measured optical center G1, 44 mas from the VLBI source $\mathrm{G}^{\prime}$, and 64 mas from the VLA source G. Clearly this separation would have been prohibitive if we had used the 1 mas error bars of the radio sources as the uncertainty in G1 center of mass position.

We experiment with forcing the galaxy center of mass to be coincident with $\mathrm{G}^{\prime}$ to the 1 mas precision of VLBI. This was the procedure of FGS in their analysis. In this case the SPLS model fits very poorly to the data, never obtaining a $\chi^{2}$ below $442\left(\bar{\chi}^{2}>73\right)$. The contribution to the overall $\chi^{2}$ from the magnification tensor constraints only marginally increases (to 22.7, up from 21.5), with essentially the entire extra $\chi^{2}$ coming from badly fit image positions. Our model has the most difficulty in simultaneously fitting both $\left(\mathrm{G}^{\prime}-B_{1}\right)_{x}$ and $\left(B_{5}-B_{1}\right)_{x}$, which are each almost 13 standard errors from the VLBI measured values and account for two-thirds of the image position $\chi^{2}$. The FGS model (see $\S 5.2$ ) fares better, but still has an unacceptably large $\bar{\chi}^{2}$ value of 43 . Adding a compact nucleus to the SPLS (see \$5.3) achieves similar results, $\bar{\chi}^{2}=48$. In both cases the $\chi^{2}$ contribution from magnification constraints is $60 \%$, as compared with $(22.7 / 442) \approx 5 \%$ for the basic SPLS.

\subsubsection{Fitted SPLS Model Parameters}

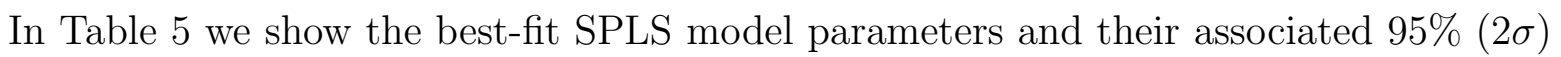
confidence limits. Because of our poor $\bar{\chi}^{2}$, we have estimated the $2 \sigma$ bounds such that they correspond to $\Delta \chi^{2}=4 \bar{\chi}^{2}$ rather than $\Delta \chi^{2}=4$.

Of particular interest are the limits on $\theta_{c}$ and $\eta$. The best-fit core radius is zero, and models with core radii in excess of $0^{\prime \prime} .11$ are excluded at the $2 \sigma$ level. At the lens redshift of 0.36 , this corresponds to a $2 \sigma$ upper limit of $330 h^{-1}$ pc on the linear core radius of the lensing galaxy. The limit on $\theta_{c}$ arises primarily from the limit on the flux of the third image 
( $c f$. Wallington \& Narayan 1993). The predicted third image flux increases rapidly with core radius, exceeding the $5 \sigma$ detection limit of $\left\|M_{C B}\right\|<1 / 30$ for $\theta_{c} \geq 63$ mas, or $r_{c} \geq 190 h^{-1}$ pc.

The best-fit value of the radial power law index $\eta$ is $\approx 1.16$, which makes the density profile of the lens slightly shallower than isothermal $(\eta=1)$. If $\eta$ is pushed lower, the model compensates by raising the core radius. This is only effective down to $\eta \approx 1.10$, at which point the core radius becomes large enough that the third image flux exceeds the $5 \sigma$ detection limit. Because of this, an isothermal profile is ruled out quite strongly. Our best "isothermal" model has $\Delta \chi^{2}=84.3$, which is unacceptably large. In the other direction, we find that the fit degrades rapidly for $\eta>1.17$, with $\eta>1.20$ excluded at the $6 \sigma$ level.

We illustrate the bounds on $\theta_{c}$ and $\eta$ in Figure 1, where we display contours of constant $\chi^{2}$ corresponding to best-fit models with fixed values of these two parameters. The most striking feature of the figure is the fact that the range of models which fall within the $2 \sigma$ contour is limited to a narrow valley covering quite a small range of the two parameters. This is despite the fact that we have conservatively defined the $2 \sigma$ limit as $\Delta \chi^{2}=4 \bar{\chi}^{2}$. In other words, the $0957+561$ observations do an excellent job of constraining the parameters of our mass model despite the large $\chi^{2}$ for the best-fit. Even fairly small deviations in the model parameters about their optimal values give a far worse fit to the data. The reason is that many of the data constraints have extremely small quoted errors so that the model has to be just right even to fit within $2 \sigma$. The large correlations between the magnification matrix elements quoted by G94 (Table 1) make the problem more acute.

Note from Figure [1 that the best-fit model has $\theta_{c}=0$ and therefore lies at one edge of the allowed parameter space. Because the surfaces of constant $\chi^{2}$ are truncated at this edge, we do not have gaussian-distributed uncertainties for our parameter estimates. Generally, most of the model parameters are tightly constrained to one side of their best-fit value and much less constrained in the opposite direction, corresponding to the narrow $\chi^{2}$ "valley" seen in Figure 11. Despite the non-gaussian errors, we have chosen to use $\Delta \chi^{2}=4 \bar{\chi}^{2}$ as our definition of the "95\%" confidence limits for the parameters. These are the values listed in Table 5 .

\subsubsection{Implications for $H_{0}$}

Given a set of fitted model parameters, we can estimate the dimensionless Hubble parameter $h_{1.5}$ as described in $\$ 4.4$. Figure 2 shows contours of $h_{1.5}$ overlaid on contours of $\chi^{2}$. We see that the $h_{1.5}$ contours are roughly parallel to the long axis of the valley of good solutions. Because of this, only a narrow range of values of $h_{1.5}$ is allowed. 
We quantify the limits on $h_{1.5}$ as follows. Given a parameter set $\mathbf{p}$ with corresponding goodness-of-fit $\chi^{2}(\mathbf{p})$ and derived Hubble parameter $h_{1.5}(\mathbf{p})$, we solve for the function $\chi^{2}\left(h_{1.5}\right)$ via the method of Lagrange multipliers. We obtain points on the $\chi^{2}\left(h_{1.5}\right)$ curve by finding the parameter set $\mathbf{p}$ which minimizes the function

$$
F(\mathbf{p} ; \lambda) \equiv \chi^{2}(\mathbf{p})+\lambda h_{1.5}(\mathbf{p})
$$

for various values of the Lagrange multiplier $\lambda$. Each choice of $\lambda$ generates a pair of values for $\chi^{2}$ and $h_{1.5}$ which represent the least $\chi^{2}$ for the subset of parameter combinations which yield the given $h_{1.5}$. The results of this procedure are shown in Figure 3. The best-fit model gives $h_{1.5}=0.605$, with the $2 \sigma$ interval given by $0.583<h_{1.5}<0.658$. Substituting this value into equation [4-14], we then obtain

$$
\begin{aligned}
H_{0} & =\left(60.5_{-2.2}^{+4.3} \mathrm{~km} \mathrm{~s}^{-1} \mathrm{Mpc}^{-1}\right)(1-\kappa)\left(\frac{1.5 \mathrm{yr}}{\Delta \tau_{B A}}\right) \\
& =\left(82.5_{-3.0}^{+5.9} \mathrm{~km} \mathrm{~s}^{-1} \mathrm{Mpc}^{-1}\right)(1-\kappa)\left(\frac{1.1 \mathrm{yr}}{\Delta \tau_{B A}}\right) .
\end{aligned}
$$

\subsection{Testing the FGS Model}

We have also tested the family of models used by FGS and described in $\$$ t.2. We obtain a best-fit $\chi^{2}$ of 28.4 , corresponding to $\bar{\chi}^{2}=28.4 / 5=5.7$. This is significantly worse than the $\bar{\chi}^{2}=4.3$ fit we obtained with the SPLS model.

In Table 6 we list the best-fit parameter values we obtain with the FGS model, and compare these with the values previously obtained by FGS using their more limited data. For almost all parameters, our new estimates deviate significantly from the old ones. This is somewhat worrisome since it suggests that this lens model may not be very robust. Incidentally, if we use the original model parameters as given by EGS with our data constraints, we obtain an extremely poor $\chi^{2}$ of $3.3 \times 10^{4}$.

Our estimate for $h_{1.5}$ from the FGS models is 0.732 , with $95 \%$ confidence limits given by $0.658<h_{1.5}<0.795$. The $2 \sigma$ lower limit obtained here overlaps the $2 \sigma$ upper limit of the SPLS model and therefore the two models may be considered to be marginally consistent. However, between the two, the models span quite a wide range of $h_{1.5}$. This is disturbing since it suggests that the data are still unable to constrain the lens model very well. BTK made a similar point with a smaller data set. Another disturbing feature is that the value of $h_{1.5}$ suggested by our current best-fit FGS model differs considerably from the value $h_{1.5} \approx 0.60$ which FGS obtained with their orginal fits. This is a reflection of the large changes in the parameters of the model as shown in Table 6. Once again it implies that the FGS model is 
not well-constrained. One odd feature of the FGS model which must be mentioned is the extremely massive compact nucleus required with this model. At a mass of 110 billion $M_{\odot}$ $\left(110 M_{9}\right)$, this compact nucleus of the model is unlikely to represent a supermassive black hole at the center of the galaxy. It is unclear what this mass represents.

\subsection{Results for SPLS Models with Compact Nucleus}

Because EGS found that including a compact nucleus at the center of G1 greatly improved their fit to the data, we have tested modified SPLS models where we add a compact central nucleus of mass $M_{\mathrm{bh}}$. For the expanded SPLS models with compact nucleus we find that the best fit model has $M_{\mathrm{bh}}=27 M_{9}$ and $\chi^{2}=22.1$, for $\bar{\chi}^{2}=22.1 / 4=5.5$. This reduced $\chi^{2}$ is similar to the $\bar{\chi}^{2}=5.7$ we obtain with the FGS model-fitting. We see that both models with a G1 compact nucleus do a significantly worse job than the basic SPLS. In Table 7 we list the parameter values of the best-fit SPLS with a compact nucleus. These may be compared with the parameters of the basic SPLS model $\left(M_{\mathrm{bh}} \equiv 0\right)$ listed in Table 5. We see that $\eta$ has increased from 1.16 to 1.38 , so that the deviation from isothermality is larger. Most of the other parameters are almost unchanged. The derived Hubble parameter is significantly smaller, however, at $h_{1.5}=0.502$.

As we increase the mass of the compact nucleus beyond the biest-fit $27 M_{9}$ while optimizing the remaining parameters, we find that the $\chi^{2}$ increases very slowly, not reaching $\Delta \chi^{2}=\bar{\chi}^{2}$ until $M_{\mathrm{bh}} \approx 110 M_{9}$. Over this range, the core radius steadily rises and the powerlaw exponent drops toward zero. Beyond $M \approx 118 M_{9}$ the $\chi^{2}$ becomes worse very quickly. At $M_{\mathrm{bh}} \approx 125 M_{9}$, the model can do no better than $\chi^{2}=100$. The transition happens at the point where the Einstein radius $\alpha_{\mathrm{bh}}$ of the nucleus becomes comparable to the angular separation between G1 and B. Models with more massive nuclei have great difficulty fitting the $\mathrm{B}$ image and therefore give large $\chi^{2}$. Near the limiting mass, the core radius of the galaxy becomes large, comparable to the G1-B separation, and the index $\eta$ approaches zero.

Table 7 gives parameter values corresponding to a few specially selected SPLS models with compact nucleus. In addition to the best-fit model which we have already discussed, we show the best-fit isothermal $(\eta=1)$ model, where $M_{\mathrm{bh}}=78.8 M_{9}$, and an FGS-like SPLS where we fix $M_{\mathrm{bh}}$ equal to the optimum value obtained with the FGS model. Generally, we find that up to $M_{\mathrm{bh}} \sim 50 M_{9}$, the core radius is zero and $\eta$ increases. Above this mass, $\eta$ starts decreasing and the core radius goes up. The variations are particularly rapid as $M_{\mathrm{bh}}$ approaches the limiting value. 
The derived values of the Hubble parameter shown in Table 7 reveal a similar behavior. Until $M_{\mathrm{bh}} \sim 50 M_{9}, h_{1.5}$ decreases, going down to about 0.5. For more massive nuclei, $h_{1.5}$ turns around, increasing almost to 0.75 near the FGS mass. An interesting result is that the value of $h_{1.5}$ we obtain with the modified SPLS model is very similar to that with the FGS model at the same mass of the nucleus. Therefore, the question of whether or not to take seriously the value of $h_{1.5}=0.75$ obtained with the FGS model boils down to whether or not a nucleus with a mass of 110 billion $M_{\odot}$ is reasonable. We ourselves find this mass uncomfortably large. A more reasonable nucleus of a few billion $M_{\odot}$, corresponding say to an AGN-like supermassive black hole, gives results very similar to those of the basic SPLS model described in $\$ 5.1$ and therefore suggests $h_{1.5} \sim 0.6$.

\subsection{Considering an Elliptical G1 Mass Distribution}

All of the above models for the $0957+561$ lens system assume azimuthal symmetry of the galaxy G1. While azimuthal symmetry is expedient for calculations, there is evidence even in the early observations of G1 by Young et al. (1980) that the galaxy has elliptical isophotes. BTK disputed the Young et al. ellipticity measurement of $e=0.13$, suggesting that contamination from the inner quasar image led to an underestimate of the true ellipticity. From their own observations, BTK reported a more substantial ellipticity $e \approx 0.30$. Both studies agreed that the position angle of the G1 major axis is $55^{\circ}$ east of north. If the G1 mass distribution is as flattened as the isophotes, one might worry that the poor $\bar{\chi}^{2}$ of the various models discussed above stems from their common assumption of G1 azimuthal symmetry. In addition, we would like to be assured that the derived $H_{0}$ for the SPLS model is not significantly affected if the G1 mass distribution includes some ellipticity.

We address these concerns by fitting the data to an elliptical variant of the SPLS. The most straightforward adaptation of our spherical mass distribution to an elliptical mass distribution replaces equation [4-3] by

$$
\Sigma(\xi, \varphi)=\Sigma_{0}\left\{1+\frac{\xi^{2}}{r_{c}^{2}}\left[1-\epsilon \cos 2\left(\varphi-\varphi_{\epsilon}\right)\right]\right\}^{(\eta-2) / 2}
$$

where $\varphi$ is the position angle on the sky with respect to the center of G1. The circular isodensity contours of the SPLS are now replaced with concentric ellipsoids having common asphericity parameter $\epsilon$ and major-axis position angle $\varphi_{\epsilon}$. We refer to this model as the softened power-law homoeoid (SPLH). In order not to introduce additional free parameters into our fitting, we fix the ellipticity and position angle of the SPLH to match the isophotal ellipticity and position angle of G1 as observed by BTK. 
Unfortunately, there are very few instances in which the deflection function for elliptical mass distributions can be expressed in closed form. Kassiola \& Kovner (1993, hereafter KK) pointed out that the softened isothermal $(\eta=1)$ homoeoid is one such instance in which the lensing properties are analytic. We have found that the deflection function for singular $\left(\theta_{c}=0\right)$ power-law homoeoids may also be expressed in closed form. In $\$$ A.1 we provide a more detailed discussion of the lensing properties of these particular cases. As we noted in $\S 5.1 .1$, the introduction of an adjustable radial power-law index $\eta$ is the distinctive feature of our mass model that allows us to fit the $0957+561$ system without a supermassive black hole and with a much improved $\chi^{2}$ over forced-isothermal models. We therefore hesitate to restrict ourselves to an isothermal homoeoid, as do Kormann, Schneider, \& Bartelmann (1994) in their unsuccessful attempt to model the gravitational lens producing the quadruple image B1422+231. We also do not wish to restrict ourselves to only singular elliptical power-law mass distributions because we have seen that the data accomodate finite-core SPLS models ( $c f$. Fig. (1), even if our best-fit SPLS has a vanishing core radius.

The only alternative that allows us to work with lens equations in closed form is to approximate the SPLH with a suitable elliptical potential model. As we show in $\S A .2$, the tilted Plummer family of elliptical potentials possesses the appropriate parameters to approximate the behavior of the SPLH. The approximation is quite good for the $0957+561$ system because we are fitting G1 with a core radius much smaller than the galaxy-image separations, and we assume that the mass distribution is no more elliptical than the observed isophotal ellipticity $e \lesssim 0.30$ mentioned above. KK have shown that for such small ellipticity, an elliptical potential is a very accurate representation of the true potential of an elliptical mass distribution. We refer the reader to $\S$ A.2 for a detailed explanation of the tilted Plummer potential approximation.

Taking the G1 mass distribution to be as flattened as the surface brightness, $e=0.30$, we find that the SPLH fits slightly better than the SPLS. We summarize the results of our model-fitting with an elliptical G1 in Table 8. The SPLH gives $\bar{\chi}^{2}=3.8$ in contrast to $\bar{\chi}^{2}=4.3$ for the SPLS, both with six degrees of freedom. Almost all of the $\chi^{2}$ reduction comes from a better fit to the observed $(G 1-B)_{y}$. The elliptical G1 does not help the problematic $\dot{M}_{2}$, which still remains 1.8 standard deviations below the value observed by G94. Comparing Table 8 with Table 5, we see that adding ellipticity to the G1 density profile makes little change to the galaxy mass model parameters. In particular, the elliptical model best-fit core radius $\theta_{c} \ll 1$ mas, Einstein radius $\alpha_{E}=2$ ". 51 , and power-law exponent $\eta=1.157$ are all well within the $2 \sigma$ confidence limits of the SPLS fitting. While the bestfit scaled shear remains exactly the same at $\gamma^{\prime}=0.224$, we find that the optimal shear position angle rotates substantially to $\phi=-76.9$. This value is far outside the $2 \sigma$ bounds $-65^{\circ} .1<\phi<-63.3$ we obtain when fitting to the SPLS. 
These results are not entirely surprising, as we might expect the shift to an elliptical G1 to have the most impact upon the fitted external shear and position angle. Just as $\gamma$ compensates for the shear induced by the cluster, so too does it compensate for shear caused by the lensing galaxy and not adequately reproduced by a SPLS model. Now introducing a separate ellipticity due to G1, we find that the external shear adjusts so as to model only the cluster shear, but to first order none of the G1 parameters are affected. Of course, we have assumed that the mass ellipticity of G1 is the same as its isophotal ellipticity. If the mass is significantly more distorted, then we expect bigger changes.

Despite the significant shift in external shear direction, the best-fit elliptical G1 model gives a Hubble parameter of $h_{1.5}=0.616$. This is a deviation of less than two percent from the $h_{1.5}=0.605$ we obtained with the SPLS, and well within the $2 \sigma$ SPLS confidence interval of $0.583<h_{1.5}<0.658$. We therefore conclude that asphericity of the mass in G1 does not significantly alter the results we have obtained by fitting an SPLS, particularly with regard to the derived Hubble constant.

\subsection{Considering Perturbations to the Cluster Model}

Up to this point, all of our $0957+561$ lens models have assumed that we may add to the potential of G1 a locally quadratic potential due to the cluster, characterized by fixed convergence, shear, and shear position angle. As we have seen in $\$ 5.4$, this "external" quadratic potential may also compensate for failings in our particular choice of the G1 mass distribution. Even if we were to have chanced upon the perfect parameterization of the G1 potential, we may nonetheless obtain a poor match to the observations if there were too much "clumpiness" in the local cluster potential around G1 for our quadratic external potential to handle. This possibility is heightened by the large $\left(\approx 6^{\prime \prime}\right)$ separation between the two images of $0957+561$. Assuming for the moment that the cluster dark matter potential is locally quadratic about G1, we may then ask if there are other galaxies sufficiently close to G1 that their differential lensing properties from image A to image B might cause problems.

Angonin-Willaime, Soucail, \& Vanderreist (1994) surveyed the 0957+561 region, obtaining photometry complete to $R=24$ in a 4.5 field as well as spectroscopy of 34 galaxies in a $6^{\prime}$ field. Their galaxy redshifts confirmed the existence of a cluster at mean redshift $\bar{z}=0.355$ containing G1, which had been suggested by earlier redshift measurements from Garrett, Walsh, \& Carswell (1992). From a sample of 21 member galaxies, Angonin-Willaime et al. concluded that the cluster containing G1 is extended and poor, with a large (>50\%) spiral fraction. Of that sample, only two galaxies (numbered 20 and 21 in Table 2 of AngoninWillaime et al. 1992) are located within 30" of either $0957+561$ A or B. Both galaxies, here- 
after referred to as G20 and G21, are faint ellipticals at redshift $z=0.355$. Both are also sufficiently near the quasar images for concern about their contribution to higher-order terms in the expansion of the potential about G1. The nearest of the two is G20, with magnitude $R=20.69$ and offset from $0957+561 \mathrm{~B}$ of $\Delta x=77^{\prime \prime} 69, \Delta y=2$.'91. G21 has magnitude $R=21.37$ and $0957+561 \mathrm{~B}$ offset of $\Delta x=11^{\prime \prime} .05, \Delta y=-2.55$.

In order to test what effect these nearby galaxies might have on our results, we include them as singular isothermal spheres. The singular isothermal sphere is an attractive candidate not only because of its simple lensing properties, but also because of its relatively slow density falloff at large radii. This allows us to be conservative in estimating the perturbative effect of G20 and G21 on the lensing at G1. To assign isothermal velocity dispersions $\sigma$ to the galaxies, we use the Faber-Jackson relation $L=L_{*}\left(\sigma / \sigma_{*}\right)^{n}$ (Faber \& Jackson 1976). Following Kochanek (1993) we adopt the values $n=4$ and $\sigma_{*}=245 \mathrm{~km} \mathrm{~s}^{-1}$ appropriate for E/S0 galaxies. As noted by Kochanek, $245 \mathrm{~km} \mathrm{~s}^{-1}$ is at the high end of the estimated $\sigma_{*}$ range of $183-248 \mathrm{~km} \mathrm{~s}^{-1}$ from dynamical estimates. Therefore we are being still more conservative with regard to the possible degree of lensing perturbation from the nearby galaxies. We arrive at isothermal velocity dispersions of $193 \mathrm{~km} \mathrm{~s}^{-1}$ for $\mathrm{G} 20$ and $165 \mathrm{~km} \mathrm{~s}^{-1}$ for G21. These may be compared with the G1 value of $330 \mathrm{~km} \mathrm{~s}^{-1}$ given in FGS, also obtained with the Faber-Jackson relation. The corresponding Einstein radii are 0'.646 for G20 and 0'472 for G21. We point out that these Einstein radii are small fractions of the galaxies' distances from the 0957+561 images - less than 10\% for G20 and less than 5\% for G21.

We summarize in Table 8 the results of our model-fitting with a perturbed cluster. The additional galaxies bring the reduced chi-square of the fit down to 3.4, a significant improvement over the original SPLS and slightly superior to the SPLH. Better fitting of the quasar image positions is responsible for almost all of the $\chi^{2}$ reduction, as is the case in our study of G1 ellipticity (see \$5.4). Similarly, we find little improvement in either the overall magnification $\chi^{2}$ or the persistent discrepancy between model-predicted and observed $\dot{M}_{2}$.

The best-fit G1 Einstein radius and cluster scaled shear are both down by $\sim 10 \%$. The reduction of the Einstein radius is because the the two external galaxies contribute an effective mass density, or convergence $\kappa$, in the vicinity of the lensed images. As we have already discussed in $\$ 4.3$, any external $\kappa$ leads to a corresponding reduction in the mass of the primary galaxy G1. The magnitude of the shear decreases partly for the same reason and partly because the external galaxies themselves produce some of the shear needed to explain the geometry of $0957+561$. The shear position angle rotates by a significant amount (to -61.0), again indicating that the external galaxies have absorbed a fraction of the required shear. The G1 core radius and power-law index show no significant change, implying that these parameters are quite robust. 
The best-fit Hubble parameter $h_{1.5}$ is 0.582 , a drop of some $4 \%$ from the 0.605 value obtained using the plain SPLS. This shift is compatible with the $2 \sigma$ uncertainty we quoted for the SPLS model. The fact that $H_{0}$ goes down rather than up is easily explained by the fact that the two external galaxies have introduced an effective convergence into the model (see $\$ 4.3$ ). In conclusion, there are no qualitative surprises from these calculations. Quantitatively, we find that the two nearest galaxies in the cluster have insignificant effect on our estimate of the Hubble constant.

\section{Eliminating the Cluster Degeneracy}

The focus to this point has been to show how recent VLBI observations of the lensed images $0957+561 \mathrm{~A}$,B limit the range of possible lens mass models, thereby limiting the model-dependent uncertainty in the determination of $H_{0}$ using this system. Quantitatively, the result is expressed in the bounds on the Hubble parameter $h_{1.5}$ given in the previous section. In order to obtain $H_{0}$, we see from equation 4-14 that we need a measurement of the relative time delay $\Delta \tau_{B A}$ and a determination of the cluster convergence $\kappa$. Considerable work has gone into the former (e.g. Vanderreist et al. 1989; Schild 1990; Lehár et al. 1992; Press et al. 1992;; Schild \& Thomson 1993; Pelt et al. 1994; Pelt et al. 1995), and it is only a matter of time before a precise $( \pm 2 \%)$ value of $\Delta \tau_{B A}$ will be settled upon for $0957+561$. Constraining the cluster convergence is a more difficult problem. As described in $\$ 4.3$, the factor $(1-\kappa)$ in the $H_{0}$ equation $4-14$ cannot be eliminated purely by observations of the lensed images. However, as Falco et al. (1991) showed, it is possible to estimate this factor by measuring the velocity dispersion of the lensing galaxy. We apply this method in $\$ 6.1$ below. Alternatively, a measurement of the velocity dispersion of the cluster may be used $(\S 6.2)$.

\subsection{Velocity Dispersion of the Lensing Galaxy G1}

The Falco et al. (1985) degeneracy arises because all image observables are unchanged if the lensing galaxy mass is lowered by a factor $(1-\kappa)$ and replaced by a mass sheet with convergence $\kappa$. However, when such a transformation is made, the velocity dispersion of the galaxy will be lower than in the original model since the galaxy now has less mass. Turning this around, a measurement of the velocity dispersion of the lensing galaxy allows us to normalize the lens mass model and thereby constrain $\kappa$. Because the mass of the galaxy scales linearly with the square of the line-of-sight velocity dispersion, $\left\langle v_{\text {los }}^{2}\right\rangle$, we may rewrite 
equation 4-14 as

$$
H_{0}=\left(100 h_{1.5} \mathrm{~km} \mathrm{~s}^{-1} \mathrm{Mpc}^{-1}\right)\left(\frac{\left\langle v_{\mathrm{los}}^{2}\right\rangle_{\mathrm{obs}}}{\left\langle v_{\mathrm{los}}^{2}\right\rangle_{\mathrm{mod}}}\right)\left(\frac{1.5 \mathrm{yr}}{\Delta \tau_{i j}}\right),
$$

where $\left\langle v_{\text {los }}^{2}\right\rangle_{\text {mod }}$ is the expected velocity dispersion of the model lens galaxy in the limit that the surrounding cluster has zero convergence.

Falco et al. (1991) applied this method to obtain their result of

$$
H_{0}=\left(60 \mathrm{~km} \mathrm{~s}^{-1} \mathrm{Mpc}^{-1}\right)\left(\frac{\sigma_{v}}{390 \mathrm{~km} \mathrm{~s}^{-1}}\right)^{2}\left(\frac{\Delta \tau_{B A}}{1.5 \mathrm{yr}}\right)^{-1} .
$$

Our analogous result from fitting the FGS model to the new VLBI data $(\S 5.2)$ is

$$
H_{0}=\left(73 \mathrm{~km} \mathrm{~s}^{-1} \mathrm{Mpc}^{-1}\right)\left(\frac{\sigma_{v}}{341 \mathrm{~km} \mathrm{~s}^{-1}}\right)^{2}\left(\frac{\Delta \tau_{B A}}{1.5 \mathrm{yr}}\right)^{-1} .
$$

In practice, one does not measure the dark matter velocity dispersion $\sigma_{v}$, but rather the velocity dispersion $\left\langle v_{\text {los }}^{2}\right\rangle$ of the luminous matter in the lensing galaxy, which need not be equal to $\sigma_{v}^{2}$. Thus it is dangerous simply to take a measured G1 velocity dispersion and substitute it for $\sigma_{v}^{2}$ in the above equations.

In the following calculations with the SPLS model, we use the virial theorem to estimate the model stellar velocity dispersion $\left\langle v_{\text {los }}^{2}\right\rangle_{\text {mod }}$ directly, for use in equation [6-1]. For notational convenience we define $\sigma^{2} \equiv\left\langle v_{\text {los }}^{2}\right\rangle$. In order to obtain a dispersion estimate that most directly compares with observation, our calculations below account for possible anisotropic orbits of the stars in the galaxy and also for the finite aperture of the slit used in the velocity dispersion measurements.

By taking a suitable moment of the Jean's equation, Kochanek (1993) has shown that the line-of-sight velocity dispersion of the stars in a spherical galaxy satisfies

$$
\sigma_{\text {mod }}^{2}=\left(\frac{G}{3}\right) \frac{\int_{0}^{\infty} r \nu(r) M(r) d r}{\int_{0}^{\infty} r^{2} \nu(r) d r},
$$

where $M(r)$ is the enclosed total mass at radius $r$, and $\nu(r)$ is the volume luminosity density. Note that equation 6-2] explicitly allows for the possibility that the luminous matter may have a different distribution than the total mass. For the SPLS lens model, $M(r)$ is given by

$$
\begin{aligned}
M(r) & =4 \pi \int_{0}^{r} r^{\prime 2} \rho\left(r^{\prime}\right) d r^{\prime}=4 \pi \rho_{0} \int_{0}^{r}\left(1+\frac{r^{\prime 2}}{r_{c}^{2}}\right)^{(\eta-3) / 2} r^{\prime 2} d r^{\prime} \\
& =\frac{4 \pi}{3} \rho_{0} r_{2}^{3} F_{1}\left(\frac{3}{2}, \frac{3-\eta}{2}, \frac{5}{2} ;-\frac{r^{2}}{r_{c}^{2}}\right)
\end{aligned}
$$


where ${ }_{2} F_{1}(a, b, c ; z)$ is the hypergeometric function ( $c f$. Abramowitz \& Stegun 1964). We numerically evaluate ${ }_{2} F_{1}(a, b, c ; z)$ by making use of the fact that the function solves the hypergeometric differential equation

$$
z(1-z) \frac{d^{2} F}{d z^{2}}=a b F-[c-(a+b+1) z] \frac{d F}{d z} .
$$

To obtain $\nu(r)$ we start with the observed surface brightness profile of G1, which is well-fit by a de Vaucouleurs profile with radius $R_{e}=4^{\prime \prime} .5 \pm 0$ '.64 (BTK). We then take $\nu(r)$ to be the spherically symmetric function which produces the de Vaucouleurs (1948) surface brightness function $I(R)=I_{e} \exp \left\{-7.67\left[\left(R / R_{e}\right)^{1 / 4}-1\right]\right\}$. We compute $\nu(r)$ via Abel inversion (it cf. Binney \& Tremaine 1987) of the de Vaucouleurs profile $I(R)$ according to

$$
\nu(r)=-\frac{1}{\pi} \int_{r}^{\infty} \frac{d I(R)}{d R} \frac{d R}{\sqrt{R^{2}-r^{2}}}
$$

Two further issues need to be considered before using equation [6-2] to estimate $\sigma_{\text {mod }}^{2}$. First, the derivation of equation [6-2] assumes that the entire luminosity distribution is sampled - the integrals of equation [6-2] are evaluated out to infinite radius from the galaxy center. In practice, a velocity dispersion measurement is taken through a narrow-slit mask, and thus gives disproportionate weighting to the central region of the galaxy luminosity distribution. Kochanek (1993) addresses this issue and gives a corrected form of equation 621 taking into account finite-aperture effects. We have applied Kochanek's method assuming that the velocity dispersion of the galaxy is measured with a long slit of angular width $1^{\prime \prime}$. The second issue concerns the degree of isotropy of the stellar orbits. Whereas equation 6-2 when integrated out to infinity is valid regardless of the shapes of the orbits, the predicted dispersion measured through a finite aperture varies with orbit anisotropy. Kochanek (1993) has considered this issue as well and has presented results for a stellar system with a constant anisotropy factor, $q$, relating radial and tangential velocity dispersions $\left(\sigma_{r, \theta, \phi}^{2}\right)$ of orbits at any given radius:

$$
\sigma_{\theta}^{2}(r)=\sigma_{\phi}^{2}(r)=(1-q) \sigma_{r}^{2}(r)
$$

Assuming isotropic $(q=0)$ orbits in G1 and a $1^{\prime \prime}$ slit aperture, we find that the bestfit SPLS model without compact nucleus (Table 5) predicts $\sigma_{\text {mod }}=321.7_{-2}^{+3} \mathrm{~km} \mathrm{~s}^{-1}$, with 95\% confidence limits. We show in Figure 1 a map of the predicted velocity dispersion as a function of core radius and power-law exponent. From this plot we see that contours of constant $\sigma_{\text {mod }}$ are remarkably parallel to the contours of $\chi^{2}$, so that $\sigma_{\text {mod }}$ varies by only $\sim \pm 1 \%$ over the range of allowed lens model parameters. A somewhat larger uncertainty is present if we allow for anisotropic orbits. Figure 5 shows $\chi^{2}$ versus $\sigma_{\text {mod }}$ corresponding to three values of $q$ : $-0.2,0,0.2$. We find that the shape of the curve is virtually unchanged 
over this range of anisotropy, but the best-fit velocity scales approximately as $(1-q)^{0.14}$. If we assume that the bright cluster elliptical G1 has $|q|<0.2$, the fractional uncertainty in $\sigma_{\text {mod }}^{2}$ due to uncertainty in $q$ is no more than $6 \%$. This is reassuring, as we expect the uncertainty in the measured dispersion to be at least this high.

Thus, we finally obtain the following result for the Hubble constant:

$$
\begin{aligned}
H_{0} & =60.5_{-4.1}^{+6.4}\left(\frac{\sigma_{\mathrm{obs}}}{322 \mathrm{~km} \mathrm{~s}^{-1}}\right)^{2}\left(\frac{1.5 \mathrm{yr}}{\Delta \tau_{B A}}\right) \mathrm{km} \mathrm{s}^{-1} \mathrm{Mpc}^{-1} \\
& =82.5_{-5.6}^{+8.7}\left(\frac{\sigma_{\mathrm{obs}}}{322 \mathrm{~km} \mathrm{~s}^{-1}}\right)^{2}\left(\frac{1.1 \mathrm{yr}}{\Delta \tau_{B A}}\right) \mathrm{km} \mathrm{s}^{-1} \mathrm{Mpc}^{-1}
\end{aligned}
$$

where we now have absorbed the model parameter uncertainty in $\sigma_{\text {mod }}^{2}$ into the error estimate on the coefficient. We have also included (in quadrature) the aforementioned $6 \%$ uncertainty due to the unknown G1 anisotropy q. Equation 6-7 shows that with good measurements of the relative time delay and of the velocity dispersion of the galaxy in $0957+561$, it is possible to obtain a comparatively precise determination of the Hubble constant. The main residual uncertainty would arise from our incomplete coverage of model space by restricting ourselves to the SPLS model. We discuss this issue in $\S 7$.

\subsection{Direct Measurement of the Cluster Potential}

In addition to measuring the velocity dispersion of G1, we may also break the degeneracy between the lensing galaxy and the cluster by making direct measurements of the surrounding cluster. One approach is to measure the core radius $\theta_{c l}$ and velocity dispersion $\sigma_{c l}$ of the cluster. Assume that the cluster potential $\phi_{c l}$ corresponds to a softened isothermal sphere with the following simple form,

$$
\phi_{c l}(\theta)=b_{c l}\left(\theta^{2}+\theta_{c l}^{2}\right), \quad b_{c l}=17^{\prime \prime} \cdot 3\left(\frac{\sigma_{c l}}{1000 \mathrm{~km} \mathrm{~s}^{-1}}\right)^{2},
$$

where $b_{c l}$ is the critical radius of the cluster. If $\zeta$ represents the angular separation of the $0957+561$ images from the cluster center, the expansion of equation 6-8] at the galaxy center then yields a local convergence $\kappa$ of the form (Kochanek 1991)

$$
\kappa=\frac{b_{c l}\left(\zeta^{2}+2 \theta_{c l}^{2}\right)}{2\left(\zeta^{2}+\theta_{c l}^{2}\right)^{3 / 2}} .
$$

This relation allows us to estimate $\kappa$ knowing the velocity dispersion of the cluster $\sigma_{c l}$, its core radius $\theta_{c l}$, and the position of the cluster center. If we take $\sigma_{c l}=600 \mathrm{~km} \mathrm{~s}^{-1}$, $\theta_{c l}=30^{\prime \prime}$, and $\zeta=25^{\prime \prime}$ as suggested by Rhee et al. (1995), we obtain $\kappa=0.13$. The present 
uncertainty in these cluster parameters places the cluster convergence in the approximate range $0.1 \lesssim \kappa \lesssim 0.2$. Equation [6-9] is valid only if the cluster is smooth and relaxed (see $\S 5.5)$.

Alternately one may try to infer the cluster potential directly through its lensing effects on background galaxies. Kaiser \& Squires (1993) showed that the shearing of background galaxies by a galaxy cluster may be inverted to recover a map of the projected cluster mass density, though subject to an overall degeneracy (Schneider \& Seitz 1995) similar to the Falco et al. (1985) degeneracy discussed above. The degeneracy can be removed by measuring magnifications of background galaxies in addition to shear distortions (Broadhurst et al. 1995: Bartelmann \& Narayan 1995). Preliminary results using a variant of the Kaiser \& Squires method have been obtained for the cluster in 0957+561 (Dahle et al. 1994; Rhee, Fischer, \& Tyson 1995) and it is hoped that more detailed information on the cluster potential will soon be available. Such studies will at the very least provide a check on whether or not the quadratic cluster potential model introduced by FGS and used in this paper is valid. An additional test would be to compare the scaled shear and its orientation as determined by our model with the direct estimates of these quantities from the cluster mass reconstruction.

\section{Summary and Discussion}

The main result of this paper is that we have developed a new and more general model of the lensing mass in the double quasar $0957+561$. The model consists of two parts: (i) a three parameter softened power-law sphere (SPLS) mass model ( $\$ 4.1$ ) for the primary lens galaxy G1, which is more general than previous models in that it allows for a variable powerlaw radial dependence, and (ii) a two-parameter model ( $\$ .3$ ) of the form proposed by FGS to describe the shear due to the surrounding cluster of galaxies. By using a larger set of data constraints than in previous analyses, and by making use especially of the recent VLBI observations of Garrett et al. (1994), we are able to constrain the parameters of our model quite tightly. The best-fit values of the five model parameters and their $95 \%$ confidence limits are shown in Table 5 .

We obtain quite a strong upper limit on the angular core radius of the galaxy. This limit is set primarily by the requirement that the model be consistent with the absence of a third image of the quasar near the center of the lensing galaxy. The limit on the linear core radius is $330 h^{-1}$ pc, which is similar to limits obtained by Wallington \& Narayan (1993) and KK. The main difference is that our result refers to a specific galaxy whereas Wallington \& Narayan and KK carried out a statistical analysis of the ensemble of lens galaxies. 
We are able to constrain the radial index $\eta$ of the lens model (cf. eq. [4-1]) to within a $10 \%$ range. The allowed range of $\eta$ is close to the isothermal value, $\eta=1$. However, exact isothermality seems to be ruled out and our model suggests that the density falls more slowly than $r^{-2}$ at large radius. The tight constraint on $\eta$ plus the fact that the model makes use of images located as far as $5^{\prime \prime}$ from the galaxy center makes this a fairly significant result. The light from the lens galaxy fits a de Vaucouleurs profile (BTK, Angonin-Willaime et al. 1994), which falls off at large radii much more steeply than a mass-traces-light isothermal distribution. Our lens model therefore indicates a substantial amount of dark matter out to at least $\sim 15 h^{-1} \mathrm{kpc}$ from the center of the lens. A similar result was obtained by Kochanek (1995) for the lensing galaxy in the radio ring source MG1654, but out to a somewhat smaller radius. Using a different approach, but again based on gravitational lensing, Brainerd, Blandford, \& Smail (1995) found evidence for isothermal halos in galaxies extending over $100 \mathrm{kpc}$ from the center. These studies illustrate the unique advantages of gravitational lensing for studying the mass distributions of distant galaxies.

In addition to the SPLS model, we also test an FGS-like model of the lensing galaxy which consists of an approximate King potential (with two parameters) plus a compact central mass. The idea here is to repeat the analysis of FGS using our larger data set to better constrain the model. The best-fit FGS parameters are shown in Table 6, and turn out to be quite different from the values published by FGS. Adding more data thus seems to have modified the model significantly, suggesting that the parameter values determined by FGS were not robust. One reason could be that FGS had only one degree of freedom in their fit. Another noteworthy feature of the FGS model is that it requires an enormous central mass of $\sim 10^{11} M_{\odot}$. Such a large mass is unlikely to be a nuclear black hole and it is not clear exactly what it represents. As a result of the large central mass, the FGS model also has a large core radius in excess of the $0957+561 \mathrm{~B}$ separation from the galaxy center. One of the advantages of the SPLS model is that it does not require a central mass. Nevertheless, in analogy with the FGS model, we try to see what would be the effect of adding a point mass to the SPLS model. The results are discussed in $\$ 5.3$ and shown in Table 7 . In brief we find that the reduced $\chi^{2}$ worsens by 1.2 when a central mass is added, which suggests that the data do not favor the inclusion of such a mass.

One troubling feature of our best-fit model is that the reduced $\chi^{2}$ is quite large, $\bar{\chi}^{2}=4.3$. Perhaps our model, despite being more general than previous ones, is still too simple to fit all the data. Incorporating reasonable perturbations due to G1 ellipticity (\$5.4) and nearby cluster galaxies (\$5.5) results in modest $\bar{\chi}^{2}$ improvement, but never do we obtain $\bar{\chi}^{2}<3.5$ for any of our SPLS variants. Efforts are under way to map the $0957+561$ cluster mass distribution using weak distortions of background galaxies (Rhee et al. 1995). These studies should help to show whether an FGS-like quadratic potential is sufficient to account for the 
cluster lensing local to G1. If our cluster model is adequate, and we simply have not hit upon the correct shape for G1, we must look to alternate mass models for the lensing galaxy. To this end, we plan to explore more general mass-traces-light models, such as the following circularly-symmetric, four-parameter model proposed by Tremaine (1995):

$$
\Sigma(R)=\Sigma_{0}\left(\frac{R}{R_{b}}\right)^{-\gamma}\left[1+\left(\frac{R}{R_{b}}\right)^{\alpha}\right]^{(\gamma-\beta) / \alpha},
$$

where $R_{b}$ is the galaxy core radius. Surface brightness profiles of many galaxies are fit by this functional form with $1 \lesssim \alpha \lesssim 2 ; 3 \lesssim \beta \lesssim 4$; and $\gamma \lesssim 0.3$.

The poor fit we find with the FGS model is also troubling. Using the FGS model, we obtain a best-fit $\bar{\chi}^{2}=5.7$, rather worse than the SPLS and much worse than the $\bar{\chi}^{2} \simeq 1.3$ quoted by FGS in their earlier fit to lower-resolution data. We are surprised that neither the SPLS nor the FGS-type family of models can accommodate the recent VLBI data of G94. Why is $\bar{\chi}^{2}$ large for all models tested? Part of the reason seems to be that some of the data are measured with extraordinary precision, e.g. the positions of the quasar jet emission blobs are known to 0.1 mas (Table 1). The models are therefore penalized even for very small errors. In fact, had we adopted the Falco et al. (1991) convention of 1 mas VLBI positioning of the G1 center of mass, both the SPLS and the FGS models would have been grossly inconsistent with the data (\$5.1.1). As noted in FGS, having the galaxy center of mass coincident with the VLBI source provides a natural explanation that the radio emission originates in the core of this bright cluster elliptical. Our current model has no explanation for this observed radio emission. Another reason for the large $\bar{\chi}^{2}$ of all our models is the large correlations quoted by G94 among the elements of the $0957+561$ relative magnification matrix and its gradient. These correlations (Table 2) cause even fairly good fits of the measurements (Table (1) to contribute large amounts to the overall $\chi^{2}$. Further VLBI observations of the jets in $0957+561$ would be desirable both to confirm the present results and to improve the quality of the constraints.

In view of the large $\bar{\chi}^{2}$ values, we have been conservative in setting confidence limits on the various parameter estimates $(\$ 5.1 .2)$ - we have taken the $95 \%$ limits to correspond with a $\chi^{2}$ increase of $4 \bar{\chi}^{2}$ rather than 4 . Despite this conservative approach, the SPLS mass model parameters are well constrained. Moreover, when we perturb the fitting, either by giving the G1 mass distribution a modest ellipticity corresponding to the observed G1 isophotes ( $\$ 5.4$ ) or by including the nearest observed cluster members explicitly in the mass model (\$5.5), we see little change in most fitted model parameters and insignificant difference in the derived Hubble constant.

The primary aim of developing a mass model for the lensing galaxy in $0957+561$ was to use the model to estimate the Hubble constant $H_{0}$. Our main result is given in equation 
[5-2]. The tight constraints that we obtain for the parameters of the lens model translate via equation 4-12 to a correspondingly tight constraint on the relation for $H_{0}$ in terms of the relative time delay $\Delta \tau_{B A}$. The coefficient in $H_{0}$ equation [5-2 has an uncertainty of only about $\pm 5 \%$. The allowed range of $H_{0}$ is asymmetric with respect to the optimum value for reasons discussed in $\$ 5.1 .3$. Note that the results quoted in this paper correspond to a flat universe $\left(\Omega_{0}=1\right)$. Our derived value of $H_{0}$ increases almost linearly with decreasing $\Omega_{0}$, with a total increase less than $10 \%$ for $\Omega_{0}=0$.

As equation 5-2 shows, $0957+561$ could be used to obtain a useful estimate of $H_{0}$ provided the relative time delay $\Delta \tau_{B A}$ is measured with sufficient precision and the convergence $\kappa$ due to the cluster is estimated. Many years of work have gone into collecting the required data for estimating $\Delta \tau_{B A}$, both in optical (Vanderreist et al. 1989; Schild 1990) and radio (Lehár et al. 1992). Estimates of $\Delta \tau_{B A}$ have however varied, with two distinct values emerging from different data sets and analyses: $\Delta \tau_{B A} \sim 410$ days (Schild \& Thomson 1993; Pelt et al. 1994; Pelt et al. 1995) and $\Delta \tau_{B A} \sim 540$ days (Press et al. 1992d). The two estimates individually have very small formal errors, so that they are seriously inconsistent with each other. Ongoing work is expected to resolve this problem shortly. Meanwhile, in this paper, we have explicitly presented our results according to both claimed time delays, using scaling factors $\left(\Delta \tau_{B A} / 1.1 \mathrm{yr}\right)$ and $\left(\Delta \tau_{B A} / 1.5 \mathrm{yr}\right)$ respectively.

The factor $(1-\kappa)$ in equation [4-14] arises because of a degeneracy in lens models discovered by Falco et al. (1985) and Gorenstein et al. (1988b). These authors showed that any lens model can be modified by reducing the mass in the lens by an arbitrary factor and substituting a constant density mass sheet of appropriate convergence $\kappa$. In such a transformation, all image observables except the time delay remain invariant. This means that a given set of lens observations cannot provide a unique mass model but rather a oneparameter family of models parametrized by $\kappa$. Since the value of $\kappa$ modifies the predicted time delay, this unfortunately means that we cannot obtain a unique estimate of $H_{0}$ from a given lens unless we independently estimate $\kappa$. Note, however, that $\kappa$ must necessarily be a positive number since it is proportional to the surface mass density of the sheet. Therefore, we can always obtain an upper bound on $H_{0}$ (Narayan 1991). From equation 5-2] we see that the $95 \%$ upper bound is $H_{0}=65 \mathrm{~km} \mathrm{~s}^{-1} \mathrm{Mpc}^{-1}$ for $\Delta \tau_{B A}=1.5 \mathrm{yr}$ and $H_{0}=88 \mathrm{~km} \mathrm{~s}^{-1} \mathrm{Mpc}^{-1}$ for $\Delta \tau_{B A}=1.1 \mathrm{yr}$.

FGS showed that it is possible to eliminate the $\kappa$ degeneracy if we could measure the line-of-sight velocity dispersion $\left\langle v_{\text {los }}^{2}\right\rangle \equiv \sigma^{2}$ of the lensing galaxy. The idea is that the family of models with different values of $\kappa$ have different amounts of mass in the primary lensing galaxy. This mass can be scaled to $\sigma^{2}$ through the virial theorem. We discuss this approach in $\$ 6.1$ and show how the method would work in the case of the SPLS model. Equation 6-7] 
gives the final result. Rhee (1991) measured $\sigma$ of the stars in the lensing galaxy in $0957+561$ to be $303 \pm 50 \mathrm{~km} \mathrm{~s}^{-1}$. Unfortunately, the measurement is not sufficiently precise to provide a significant constraint on $H_{0}$. A more precise measurement of $\sigma$ should be possible with a large optical telescope and is highly desirable, as it would eliminate this last uncertainty in the modeling of $0957+561$.

The velocity dispersion $\sigma$ refers only to the stars in the lensing galaxy, whereas the gravitational lensing is done by the total mass. There has been some uncertainty as to how the measured stellar $\sigma$ should be related to the $\sigma$ of the total mass, which is the relevant quantity for normalizing the galaxy mass distribution. The straightforward approach is to assume that the velocity dispersion of the stars and that of the dark matter particles are equal. However, Turner et al. (1985) argued that in many circumstances the $\sigma$ of the stars would be lower than that of the total mass by a factor of $(2 / 3)^{1 / 2}$. This makes quite an important difference to the results. For instance, Narayan (1991) derived on the basis of the FGS model, assuming $\Delta \tau_{B A}=536$ days (Press et al. 1992b) and $\sigma=303 \mathrm{~km} \mathrm{~s}^{-1}$ (Rhee 1991), that $H_{0}=37 \mathrm{~km} \mathrm{~s}^{-1} \mathrm{Mpc}^{-1}$ if the dark matter has the same dispersion as the stars and $H_{0}=56 \mathrm{~km} \mathrm{~s}^{-1} \mathrm{Mpc}^{-1}$ if the correction factor of $(2 / 3)^{1 / 2}$ is applied. The approach used in this paper, based on the work of Kochanek (1993), avoids the ambiguity since it is based on a fundamental application of the virial theorem. Our model here gives, for the same parameters as the ones employed by Narayan (1991), $H_{0}=55 \mathrm{~km} \mathrm{~s}^{-1} \mathrm{Mpc}^{-1}$.

Narayan (1991) has discussed an interesting additional benefit that one obtains by measuring $\sigma$. Once the mass of the lens has been normalized through such a measurement, it is possible to obtain an estimate of $H_{0}$ that is independent of the source redshift. In other words, the distance to the source drops out of the relations. This is, of course, not a particular advantage since most often the source redshift is known. However, a corollary of the theorem is that if there are additional mass sheets with shear between the lens and the source, say due to other clusters, the formula for $H_{0}$ is transparent to their presence provided the additional clusters are describable by quadratic potentials over the angular extent of the lensed images. This theorem is quite useful. There is evidence for a second cluster at redshift 0.51 in the field of 0957+561 (BTK, Angonin-Willaime et al. 1994), and there may well be other clusters at higher redshift. It is advantageous to be able to estimate $H_{0}$ independently of these complications. Another interesting result is that with a measurement of $\sigma$ one directly obtains the angular diameter distance $D_{d}$ to the lens regardless of cosmological model, i.e. the result is independent of the values of $q_{0}$ and $\Lambda$ (Narayan 1991).

The $\kappa$ degeneracy can also be eliminated by estimating the mass surface density of the cluster directly. One simple method is to measure the core radius of the cluster, its velocity dispersion, and the location of the lens relative to the cluster center. We describe 
in $\$ 6.2$ how this information can be translated into an estimate of $\kappa$. Using the parameter values given by Rhee et al. (1995), we estimate that $\kappa \sim 0.1-0.2$ for the lensing cluster in $0957+561$. This translates to $H_{0}=(47-58) \mathrm{km} \mathrm{s}^{-1} \mathrm{Mpc}^{-1}$ for $\Delta \tau_{B A}=1.5 \mathrm{yr}$ and $H_{0}=(64-80) \mathrm{km} \mathrm{s}^{-1} \mathrm{Mpc}^{-1}$ for $\Delta \tau_{B A}=1.1 \mathrm{yr}$. A more ambitious undertaking is to map the two-dimensional surface density of the cluster using the weak distortions of background galaxies. The idea for this method goes back to Tyson, Wenk, \& Valdes (1990) and Kaiser \& Squires (1993). Rhee et al. (1995) are currently applying the method to the field around $0957+561$ and results are awaited.

What is the future for lens-based measurements of the Hubble constant? We believe $0957+561$ will deliver a result soon. It is only a matter of time before the controversy over the time delay is settled. Our model ( $c f$. eq. 4-14]) will then directly provide an upper bound on $H_{0}$ or a direct estimate if we take the value of $\kappa \sim 0.1-0.2$ mentioned above. Measuring $\sigma$ and thereby obtaining a more reliable estimate of $\kappa$ is more challenging, but efforts are under way and once again we are optimistic. With measurements of both $\Delta \tau_{B A}$ and $\sigma$, our model should provide an estimate of $H_{0}$ which would be quite competitive with other determinations. There is unfortunately one major remaining uncertainty, namely whether our model captures the mass distribution of the lens sufficiently well. The poor reduced $\chi^{2}$ of our model is certainly a concern. Perhaps a mass-traces-light model will improve matters, or perhaps future observations will resolve our SPLS discrepancies. In any case, it is desirable to study additional lenses in order to obtain other independent estimates of $H_{0}$.

It is generally agreed that $0957+561$ is not the best source for estimating $H_{0}$ since the presence of the cluster adds an extra layer of complication. Several other good candidates are available where the lensing galaxy appears to be more or less isolated. Such systems are easier to model. The radio ring sources are particularly promising since the modeling of these is likely to be more reliable than with multiply-imaged optical quasars. The experience with $0957+561$ shows that having information on the full relative magnification matrix and its spatial derivative is invaluable for constraining the mass model. Therefore, resolved sources which can be mapped and modeled in detail are likely to be much superior to unresolved sources.

To conclude, we emphasize that the lens-based method of estimating $H_{0}$ is completely independent of all other methods and works directly on high redshift sources without using any intermediate distance ladders. Furthermore, the method is based on very basic geometry and physics. These are substantial advantages, and we feel that the method deserves to be pursued seriously.

We are grateful to E. Falco, M. Garrett, C. Kochanek, J. Lehár, P. Schechter, R. Schild, 
and I. Shapiro for discussions and comments, and to the referee for useful suggestions. This research was supported in part by grant AST 9423209 from the NSF.

\section{A. Approximating a Softened Power-Law Homoeoidal Lens}

\section{A.1. Lensing Properties of the SPLH}

As we often observe galaxies to have elliptical isophotes, it would be appealing to model their lensing properties with a mass distribution having elliptical isodensity contours. A simple example is the homoeoid, whose isodensity contours are concentric ellipses of constant ellipticity and position angle. The surface density of the homoeoid varies only as a function of the elliptical "radius" $r_{\mathrm{em}}$ from the center of the distribution:

$$
r_{\mathrm{em}}^{2} \equiv \frac{x^{2}}{(1+\epsilon)^{2}}+\frac{y^{2}}{(1-\epsilon)^{2}},
$$

where the parameter $\epsilon$ reflects the degree of flattening. In the above equation and throughout the remainder of this appendix, we assume that the major axis of the elliptical profile is aligned with the $x$-axis of our coordinate system. From equation [A1], we relate the asphericity parameter $\epsilon$ to the axis ratio of isodensity contours:

$$
\frac{b}{a}=\frac{1-\epsilon}{1+\epsilon}
$$

We emphasize that $\epsilon$ is not equivalent to the ellipticity $e$, defined by $e \equiv 1-(b / a)$.

Bourassa \& Kantowski (1975) found that the lensing properties of generic elliptical mass distributions may be compactly expressed by adopting a complex angular notation, where vector angles $\boldsymbol{\theta}$ map to the complex plane according to $\boldsymbol{\theta} \mapsto z=\theta_{x}+i \theta_{y}$. They derived the complex ray deflection $\alpha^{*}(z)$ for a general homoeoidal density profile $\kappa\left(r_{\mathrm{em}}\right)=\Sigma\left(r_{\mathrm{em}}\right) / \Sigma_{\mathrm{cr}}$ :

$$
\alpha^{*}(z)=2\left(1-\epsilon^{2}\right) \int_{0}^{r_{\mathrm{em}}(z)} \frac{\kappa(\rho) \rho d \rho}{S \sqrt{\bar{z}^{2}-4 \epsilon \rho^{2}}}
$$

with $\bar{z}$ as the complex conjugate of $z$. Here $S$ denotes the branch of the complex square root for which $S \sqrt{\bar{z}^{2}-4 \epsilon \rho^{2}}$ and $\bar{z}$ lie in the same quadrant of the complex plane (Bray 1984). As noted by Schramm (1994), closed solutions of equation A2 for general elliptical lenses have rarely been found (Narasimha 1982, KK) and are by construction restricted to density profiles with homoeoidal symmetry.

KK have shown that equation A2 has a closed form for softened isothermal homoeoids, 
whose density profiles are characterized by central convergence $\kappa_{0}$ and core radius $\theta_{c}$ :

$$
\kappa\left(r_{\mathrm{em}}\right)=\frac{\kappa_{0} \theta_{c}}{\sqrt{r_{\mathrm{em}}^{2}+\theta_{c}^{2}}}
$$

The complex ray deflection for the softened isothermal homoeoid is

$$
\alpha^{*}(z)=\frac{\left(1-\epsilon^{2}\right) \alpha_{\mathrm{E}}}{2 i \sqrt{\epsilon}} \ln \left\{\left(\bar{z}+2 i \theta_{c} \sqrt{\epsilon}\right)^{-1}\left[x\left(\frac{1-\epsilon}{1+\epsilon}\right)-i y\left(\frac{1+\epsilon}{1-\epsilon}\right)+2 i \sqrt{\epsilon\left(r_{\mathrm{em}}^{2}+\theta_{c}^{2}\right)}\right]\right\},
$$

where $x \equiv \operatorname{Re}(z), y \equiv \operatorname{Im}(z)$, and $\alpha_{\mathrm{E}}=2 \theta_{c} \kappa_{0}$ is the asymptotic Einstein radius for $\theta_{c}=\epsilon=0$.

We have found that a closed form of the ray deflection equation A2 also exists for the singular power-law homoeoids. The density profile of the singular power-law homoeoid is parameterized by asymptotic $(\epsilon \rightarrow 0)$ Einstein radius $\alpha_{\mathrm{E}}$ and power-law index $\eta$ of radial mass increase:

$$
\kappa\left(r_{\mathrm{em}}\right)=\frac{\eta}{2}\left(\frac{r_{\mathrm{em}}}{\alpha_{\mathrm{E}}}\right)^{\eta-2} .
$$

Substituting the surface density from equation [A5] into the integrand of equation [A2], we obtain the complex ray deflection of the singular power-law homoeoid:

$$
\alpha^{*}(z)=z\left(1-\epsilon^{2}\right)\left(\frac{r_{\mathrm{em}}}{\alpha_{\mathrm{E}}}\right)^{\eta}\left(\frac{|z|}{\alpha_{\mathrm{E}}}\right)^{-2}{ }_{2} F_{1}\left(\frac{1}{2}, \frac{\eta}{2}, 1+\frac{\eta}{2}, \frac{4 \epsilon r_{\mathrm{em}}^{2}}{\bar{z}^{2}}\right),
$$

where $|z| \equiv \sqrt{z \bar{z}}$ and ${ }_{2} F_{1}$ is the complex hypergeometric function. In the limit of azimuthal symmetry $(\epsilon=0), r_{\mathrm{em}}=|z|$ and the hypergeometric function becomes unity. Equation [A6] therefore reduces to $\alpha^{*}(z)=\left(|z| / \alpha_{\mathrm{E}}\right)^{\eta-2} z$, which may be compared with the deflection of a singular power-law sphere (eq. [4-6] with $\theta_{c}=0$ ).

\section{A.2. Tilted Plummer Elliptical Potentials}

To overcome the difficulties of elliptical mass distributions, we turn to elliptical potentials. Such potentials vary only as a function of the elliptical radius $r_{\mathrm{ep}}^{2} \equiv\left[x^{2}\left(1-\epsilon_{\mathrm{p}}\right)+\right.$ $y^{2}\left(1+\epsilon_{\mathrm{p}}\right)$ ], characterized by asphericity parameter $\epsilon_{\mathrm{p}}$. Although the lensing properties of elliptical potentials are easily expressed in closed form, their associated isodensity contours are sometimes unphysical. Elliptical potentials with more than a moderately high asphericity $\left(\epsilon_{\mathrm{p}} \gtrsim 0.2\right)$ require isodensity contours that are dumbbell-shaped ( $\mathrm{KK}$ ). In certain cases, highly flattened elliptical potentials may even require negative isodensity contours (Blandford \& Kochanek 1987). However, elliptical potentials provide quite an accurate representation of elliptical mass distributions for ellipticities $e \lesssim 0.3$. Fortunately for this study, the $0957+561$ lensing galaxy G1 has an isophotal ellipticity compatible with this limit. 
Isothermal $(\eta=1)$ elliptical potentials have been popular in the modeling of aspherical galaxies (e.g. Blandford \& Kochanek 1987; Kochanek \& Blandford 1987; Wallington \& Narayan 1993), but we seek an elliptical potential which has the radial power-law generality of the SPLH. The family of tilted Plummer potentials fits this description:

$$
\Psi=\frac{\alpha_{\mathrm{E}}^{2}}{\eta}\left[\frac{\omega_{\mathrm{p}}^{2}+r_{\mathrm{ep}}^{2}}{\alpha_{\mathrm{E}}^{2}}\right]^{\eta / 2},
$$

where the potential $\Psi$, expressed in units of $\operatorname{radian}^{2}$, is equivalent to $\psi / c^{2}$ in the notation of \&2. Here $\omega_{\mathrm{p}}$ represents the core radius of the potential, and we recycle the SPLS parameters $\alpha_{\mathrm{E}}$ and $\eta$ to represent an effective Einstein radius and radial power-law index, respectively. Taking the gradient of equation [A7], we find that the ray deflection of the tilted Plummer model is given by

$$
\left(\begin{array}{c}
\alpha_{x} \\
\alpha_{y}
\end{array}\right)=\left(\frac{\omega_{\mathrm{p}}^{2}+r_{\mathrm{ep}}^{2}}{\alpha_{\mathrm{E}}^{2}}\right)^{\frac{\eta-2}{2}}\left(\begin{array}{c}
\left(1-\epsilon_{\mathrm{p}}\right) x \\
\left(1+\epsilon_{\mathrm{p}}\right) y
\end{array}\right) .
$$

Comparing the asymptotic $\left(\omega_{\mathrm{p}}, \epsilon_{\mathrm{p}}, \theta_{c} \rightarrow 0\right)$ behavior of equation A8 with the SPLS analogue (eq. 4-6]), we see that the tilted Plummer model parameters $\alpha_{E}$ and $\eta$ and their SPLS counterparts are equivalently defined.

In order to relate the core radius parameters $\omega_{\mathrm{p}}$ and $\theta_{c}$, we follow the convention of KK, who require the equivalence of the lens central convergence $\kappa_{0}$. The Poisson equation in conjunction with equation [A7] yields the convergence for the tilted Plummer model:

$$
\begin{aligned}
\kappa\left(r_{\mathrm{ep}}\right)= & \left\{\frac{\omega_{\mathrm{p}}^{2}+x^{2}\left(1-\epsilon_{\mathrm{p}}\right)\left[(\eta / 2)\left(1-\epsilon_{\mathrm{p}}\right)+\epsilon_{\mathrm{p}}\right]+y^{2}\left(1+\epsilon_{\mathrm{p}}\right)\left[(\eta / 2)\left(1+\epsilon_{\mathrm{p}}\right)-\epsilon_{\mathrm{p}}\right]}{\omega_{\mathrm{p}}^{2}+r_{\mathrm{ep}}^{2}}\right\} \\
& \times\left(\frac{\omega_{\mathrm{p}}^{2}+r_{\mathrm{ep}}^{2}}{\alpha_{\mathrm{E}}^{2}}\right)^{\frac{\eta-2}{2}} .
\end{aligned}
$$

We obtain the convergence for the SPLH model by adding a finite core radius to the singular power-law homoeoid (eq. A3):

$$
\kappa\left(r_{\mathrm{em}}\right)=\frac{\eta}{2}\left(\frac{\theta_{c}^{2}+r_{\mathrm{em}}^{2}}{\alpha_{\mathrm{E}}^{2}}\right)^{\frac{\eta-2}{2}} .
$$

With somewhat more effort, it may also be shown that equation [A10] is the elliptical generalization $\left(r \rightarrow r_{\mathrm{em}}\right)$ of the SPLS convergence obtained from equation 44-3] divided by the

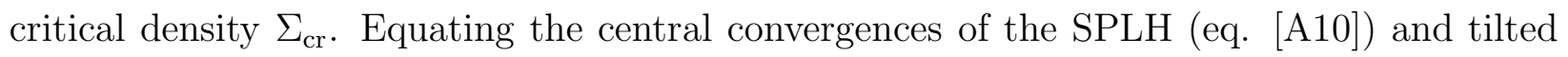
Plummer potential (eq. A9 ) gives the desired relation between the respective core radii:

$$
\omega_{\mathrm{p}}=\left(\frac{2}{\eta}\right)^{\frac{1}{2-\eta}} \theta_{c}
$$


Finally, we must determine the appropriate value for the tilted Plummer potential asphericity $\epsilon_{\mathrm{p}}$. We seek the $\epsilon_{\mathrm{p}}$ for which the axis ratio of isodensity contours at large radius, $(b / a)_{\infty}$, is equal to the observed isophotal axis ratio $(b / a) \equiv 1-e$. Making use of equation A9 in the limit $\omega_{\mathrm{p}} \rightarrow 0$, we obtain

$$
\left(\frac{b}{a}\right)_{\infty}=\left(\frac{1-\epsilon_{\mathrm{p}}}{1+\epsilon_{\mathrm{p}}}\right)^{1 / 2}\left[\frac{1-\epsilon_{\mathrm{p}}(2 / \eta-1)}{1+\epsilon_{\mathrm{p}}(2 / \eta-1)}\right]^{\frac{1}{2-\eta}}=1-e .
$$

As an aside, we note that equation A12 with $\eta=1$ simplifies to the isothermal relation noted by KK:

$$
\left(\frac{b}{a}\right)_{\infty}=\left(\frac{1-\epsilon_{\mathrm{p}}}{1+\epsilon_{\mathrm{p}}}\right)^{3 / 2} .
$$

With equations [A11] and [A12], we may now determine the set of tilted Plummer potential parameters $\left(\omega_{\mathrm{p}}, \eta, \alpha_{\mathrm{E}}\right.$, and $\left.\epsilon_{\mathrm{p}}\right)$ which approximate an arbitrary softened power-law homoeoid specified by radial parameters $\left(\theta_{c}, \eta\right.$, and $\left.\alpha_{\mathrm{E}}\right)$ and isodensity ellipticity $e$.

KK show that the tilted Plummer potential and SPLH potential approximately coincide when the asphericities and core sizes are sufficiently small. In particular, the sizes of the respective tangential caustics coincide. The G1 core sizes compatible with nondetection of a third QSO image $\left(\theta_{c} \lesssim 0^{\prime \prime} .1\right)$ are indeed small, and even at a G1 mass ellipticity of 0.30 there is only minor deviation between the models' isodensity contours ( $c f$. KK Fig. 4). We therefore conclude that our treatment of G1 as an SPLH is not compromised by approximating the lensing properties with a suitably chosen tilted Plummer potential. 
Table 1: 0957+561 Image Positions and Flux Densities from VLBI

\begin{tabular}{llcc}
\hline \hline $\begin{array}{l}\text { Emission } \\
\text { Component }\end{array}$ & $\begin{array}{l}\text { Flux Density } \\
(\mathrm{mJy})\end{array}$ & $\begin{array}{l}\text { Radius } \\
(\mathrm{mas})\end{array}$ & $\begin{array}{l}\text { Pos. Ang. } \\
\left({ }^{\circ}\right)\end{array}$ \\
\hline$A_{1}$ & $14.2 \pm 0.1$ & 0 & 0 \\
$A_{5}$ & $10.6 \pm 0.2$ & $48.3 \pm 0.1$ & $19.9 \pm 0.1$ \\
\hline$B_{1}$ & $11.4 \pm 0.1$ & 0 & 0 \\
$B_{5}$ & $7.0 \pm 0.5$ & $58.8 \pm 0.1$ & $17.8 \pm 0.1$ \\
\hline
\end{tabular}

Note. - Shown here are the two brightest pairs of emission regions identified in the six-component Gaussian model fitted by G94 to $0957+561 \mathrm{~A}$,B. The radius and position angles are given as offsets from the respective QSO central regions $A_{1}$ and $B_{1}$.

Table 2: 0957+561 Image Magnification Constraints

A. Relative Magnification Matrix Elements

\begin{tabular}{lc}
\hline \hline Quantity & Measured Value \\
\hline$M_{1}$ & $1.23 \pm 0.04$ \\
$M_{2}$ & $-0.50 \pm 0.03$ \\
$\phi_{1}\left(^{\circ}\right)$ & $18.6 \pm 0.1$ \\
$\phi_{2}\left({ }^{\circ}\right)$ & $118 \pm 6$ \\
$\dot{M}_{1}\left(10^{-3} \mathrm{mas}^{-1}\right)$ & $0.5 \pm 1.5$ \\
$\dot{M}_{2}\left(10^{-3} \mathrm{mas}^{-1}\right)$ & $2.6 \pm 0.8$ \\
\hline
\end{tabular}

B. Correlation Coefficients

\begin{tabular}{lrrrrrr}
\hline \hline Covar & $M_{1}$ & $M_{2}$ & $\phi_{1}$ & $\phi_{2}$ & $\dot{M}_{1}$ & $\dot{M}_{2}$ \\
\hline$M_{1}$ & 1.00 & & & & & \\
$M_{2}$ & 0.46 & 1.00 & & & & \\
$\phi_{1}$ & -0.39 & -0.38 & 1.00 & & & \\
$\phi_{2}$ & -0.79 & -0.61 & 0.21 & 1.00 & & \\
$\dot{M}_{1}$ & 0.96 & 0.40 & -0.22 & -0.73 & 1.00 & \\
$\dot{M}_{2}$ & 0.70 & 0.79 & -0.26 & -0.70 & 0.70 & 1.00 \\
\hline
\end{tabular}

Note. - Magnification matrix information as measured by G94 for $0957+561 \mathrm{~A}, \mathrm{~B}$. Here $M_{1,2}$ are the matrix eigenvalues from $A_{5}$ to $B_{5} ; \phi_{1,2}$ are the eigenvector position angles. $\dot{M}_{1,2}$ are spatial derivatives, taken upward along the $\mathrm{A}$ jet, of the eigenvalues of the relative magnification matrix. Also given is the normalized error covariance matrix for these values. 
Table 3: Offset of lensing galaxy center of brightness from $0957+561 \mathrm{~B}$

\begin{tabular}{|c|c|c|c|}
\hline Observation & Designation & $x$ offset & $y$ offset \\
\hline Optical $^{\mathrm{a}}$ & G1 & $0^{\prime \prime} \cdot 19 \pm 0^{\prime \prime} 03$ & $1^{\prime \prime} .00 \pm 0.03$ \\
\hline VLA $^{\mathrm{b}}$ & G. & $0^{\prime \prime} .151 \pm 0^{\prime \prime} .001$ & $1^{\prime \prime} 051 \pm 0$ 0.001 \\
\hline $\mathrm{VLBI}^{\mathrm{c}}$ & $\mathrm{G}^{\prime}$ & $0 ! 181 \pm 0.001$ & $1^{\prime \prime} 029 \pm 0^{\prime \prime} 001$ \\
\hline
\end{tabular}

a Stockton 1980; Seeing conditions better than 0."5

$b$ Roberts et al. 1985; $\lambda=6 \mathrm{~cm}$

cGorenstein et al. 1988a; $\lambda=13 \mathrm{~cm}$

Table 4: Best-Model Estimations and Goodness-of-Fit

\begin{tabular}{|c|c|c|}
\hline Observable & Model Estimate & $\frac{\text { (Obsvd. }- \text { Estd. })}{\text { Obsv. Err. }}$ \\
\hline \multicolumn{3}{|c|}{ Image Separations ${ }^{\mathrm{a}}$ : Contributed $\chi^{2}=4.5$} \\
\hline$A_{1}-B_{1}$ & $\left(-1^{\prime \prime} .25271,6^{\prime \prime} .04662\right)$ & $\left(\sim 10^{-4}, \sim 10^{-4}\right)$ \\
\hline$G 1-B_{1}$ & $\left(0^{\prime \prime} 215,1^{\prime \prime} .057\right)$ & $(0.84,1.91)$ \\
\hline$A_{5}-A_{1}$ & $(16.4,45.4)$ mas & $(0.013,0.026)$ \\
\hline$B_{5}-B_{1}$ & $(18.0,56.0)$ mas & $(0.002,-0.26)$ \\
\hline \multicolumn{3}{|c|}{ Magnifications and Gradients: Contributed $\chi^{2}=21.5$} \\
\hline$M_{1}$ & 1.244 & -0.34 \\
\hline$M_{2}$ & -0.529 & 0.95 \\
\hline$\phi_{1}$ & $18: 69$ & -0.93 \\
\hline$\phi_{2}$ & $108^{\circ}$ & 1.66 \\
\hline$\dot{M}_{1}$ & $\left(0.97 \times 10^{-3}\right) \mathrm{mas}^{-1}$ & -0.27 \\
\hline$\dot{M}_{2}$ & $\left(0.99 \times 10^{-3}\right) \mathrm{mas}^{-1}$ & 1.8 \\
\hline$\left\|M_{C B}\right\|$ & $<10^{-10}$ & 0 \\
\hline
\end{tabular}

${ }^{a}$ Expressed in the Cartesian coordinate notation $(x, y)$ described in 8 目 
Table 5: Fitted SPLS Parameters for the 0957+561 Lens System

\begin{tabular}{lcc}
\hline \hline Symbol & Best-Fit & $95 \%$ Conf. Limits \\
\hline & Lensing & Galaxy $(G 1)$ \\
\hline$\alpha_{\mathrm{E}}$ & $2^{\prime \prime} .587$ & $2^{\prime \prime} .560<\alpha_{\mathrm{E}}<2^{\prime \prime} .690$ \\
$\theta_{c}$ & $0^{\prime \prime} 00$ & $0^{\prime \prime}<\theta_{c}<0^{\prime \prime} .105$ \\
$\eta$ & 1.165 & $1.055<\eta<1.176$ \\
\hline \multicolumn{3}{c}{ External Shear } \\
\hline$\gamma^{\prime}$ & 0.224 & $0.220<\gamma^{\prime}<0.237$ \\
$\phi$ & -64.40 & $-65.13<\phi<-63.31$ \\
\hline
\end{tabular}

${ }^{a}$ Non-Gaussian, see 5.1 .1 for details

Table 6: Fitted FGS Model Parameters Compared with Previous Estimates

\begin{tabular}{lccc}
\hline \hline Paramter & Best-fit & $95 \%$ Conf. Limits & Previous Best-fit \\
\hline$\theta_{c}$ & $1^{\prime \prime} .56$ & $0^{\prime \prime} 99<\theta_{c}<2^{\prime \prime} .05$ & $2^{\prime \prime} \cdot 9 \pm 0^{\prime \prime} 1$ \\
$\sigma_{v}\left(\mathrm{~km} \mathrm{~s}^{-1}\right)$ & 340.5 & $336<\sigma_{v}<355$ & $390 \pm 4$ \\
$M_{\mathrm{bh}}\left(10^{9} M_{\odot}\right)$ & 111 & $80<M_{\mathrm{bh}}<114$ & $115 \pm 1$ \\
$\gamma^{\prime}$ & 0.273 & $0.266<\gamma^{\prime}<0.278$ & $0.18 \pm 0.01$ \\
$\phi$ & -64.9 & $-67^{\circ} 4<\phi<-622^{\circ} .1$ & $-63^{\circ} .3 \pm 0.6$ \\
\hline
\end{tabular}

${ }^{a}$ From FGS Table 3 ; variations are due to differing lensing notation conventions. 
Table 7: Results for Models with G1 Compact Nucleus

\begin{tabular}{lcccc}
\hline \hline Symbol & $\begin{array}{c}\text { Lowest } \chi^{2} \\
\text { SPLS }\end{array}$ & $\begin{array}{c}\text { Lowest } \chi^{2} \\
\text { FGS }\end{array}$ & $\begin{array}{c}\text { "Isothermal" } \\
\text { SPLS }\end{array}$ & $\begin{array}{c}M_{\mathrm{bh}}=M_{\mathrm{bh}}^{\text {FGS }} \\
\text { SPLS }\end{array}$ \\
\hline$M_{\mathrm{bh}}\left(10^{9} M_{\odot}\right)$ & 27.2 & 110.9 & 78.8 & 110.9 (fixed) \\
$\theta_{c}$ & $0^{\prime \prime} .000$ & $1^{\prime \prime} .56$ & $0^{\prime \prime} .714$ & $1^{\prime \prime} 33$ \\
$\eta$ & 1.38 & $\mathrm{~N} / \mathrm{A}$ & 1 (fixed) & 0.256 \\
$\alpha_{\mathrm{E}}$ & $2^{\prime \prime} .44$ & $\mathrm{~N} / \mathrm{A}$ & $3^{\prime \prime} .10$ & $6^{\prime \prime} .34$ \\
$\gamma^{\prime}$ & 0.194 & 0.273 & 0.238 & 0.278 \\
$\phi$ & -65.43 & -64.86 & -65.03 & $-64^{\circ} .74$ \\
\hline$\chi^{2} /$ d.f. $^{\mathrm{a}}$ & 5.5 & 5.7 & 4.9 & 5.6 \\
$h_{1.5}$ & 0.502 & 0.732 & 0.627 & 0.745
\end{tabular}

${ }^{a}$ The first model has four degrees of freedom; the rest have five.

Table 8: Results for Models with G1 Ellipticity and Perturbed Cluster

\begin{tabular}{lcc}
\hline \hline & $e=0.30$ & Perturbed Cluster \\
Symbol & SPLH $^{\mathrm{a}}$ & SPLS $^{\mathrm{b}}$ \\
\hline$\theta_{c}$ & $0^{\prime \prime} .000$ & $0^{\prime \prime} 000$ \\
$\eta$ & 1.157 & 1.159 \\
$\alpha_{\mathrm{E}}$ & $2^{\prime \prime} .50$ & $2^{\prime \prime} .33$ \\
$\gamma^{\prime}$ & 0.224 & 0.205 \\
$\phi$ & -76.95 & $-61^{\circ} .05$ \\
\hline$\chi^{2} /$ d.f. $^{\mathrm{c}}$ & 3.8 & 3.4 \\
$h_{1.5}$ & 0.615 & 0.582
\end{tabular}

${ }^{a}$ Described in $\$ 5.4$.

${ }^{b}$ Described in $\$ 5.5$.

${ }^{c}$ Both models have six degrees of freedom. 


\section{REFERENCES}

Abramowitz, M., \& Stegun, I. A. 1964, Handbook of Mathematical Functions, Applied Mathematics Series, vol. 55 (Washington: National Bureau of Standards; reprinted 1968 by Dover Publications, New York)

Angonin-Willaime, M.-C., Soucail, G., \& Vanderreist, C. 1994, A\&A, 291, 411

Bartelmann, M., \& Narayan, R. 1995, ApJ, 451, 60

Bernstein, G. M., Tyson, J. A., \& Kochanek, C. S. 1993, AJ, 105, 816

Binney, J., \& Tremaine, S. D. 1987, Galactic Dynamics (Princeton: Princeton University Press)

Blandford, R. D., \& Kochanek, C. S. 1987, ApJ, 321, 658

Blandford, R. D., \& Narayan, R. 1992, ARA\&A, 30, 311

Bonometti, R. J. 1985, Ph.D. thesis, Massachusetts Institute of Technology

Borgeest, U., \& Refsdal, S. 1984, A\&A, 141, 318

Bourassa, R. R., \& Kantowski, R. 1975, ApJ, 195, 13

Brainerd, T. G., Blandford, R. D., \& Smail, I. 1995, ApJ, in press

Bray, I. 1984, MNRAS, 208, 511

Broadhurst, T. J., Taylor, A. N., \& Peacock, J. A. 1995, ApJ, 438, 49

Dahle, H., Maddox, S. J., \& Lilje, Per B. 1994, ApJ, 435, L79

de Vaucouleurs, G. 1948, Ann.d'Ap., 11, 247

Faber, S. M., \& Jackson, R. E. 1976, ApJ, 204, 668

Falco, E. E., Gorenstein, M. V., \& Shapiro I. I. 1985, ApJ, 289, 1

Falco, E. E., Gorenstein, M. V., \& Shapiro I. I. 1991, ApJ, 372, 364

Garrett, M. A., Walsh, D., \& Carswell, R. F. 1992, MNRAS, 254, 27

Garrett, M. A., Calder, R. J., Porcas, R. W., King, L. J., Walsh, D., \& Wilkinson, P. N. 1994, MNRAS, 270, 457 
Gorenstein, M. V., Shapiro I. I., Cohen, N. L., Corey, B. E., Falco, E. E., Marcaide, J. M., Rogers, A. E. E., Whitney, A. R., Porcas, R. W., Preston, R. A., \& Ruis, A. 1983, Science, 219, 54

Gorenstein, M. V., Shapiro I. I., Rogers, A. E. E., Cohen, N. L., Corey, B. E., Porcas, R. W., Falco, E. E., Bonometti, R. J., Preston, R. A., Rius, A., \& Whitney, A. R. 1984, ApJ, 287, 538

Gorenstein, M. V., Cohen, N. L., Shapiro I. I., Rogers, A. E. E., Bonometti, R. J., Falco, E. E., Bartel, N., \& Marcaide, J. M. 1988a, ApJ, 334, 42

Gorenstein, M. V., Falco, E. E., \& Shapiro I. I. 1988b, ApJ, 327, 693

Greenfield, P. D., Roberts, D. H., \& Burke, B. F. 1985, ApJ, 293, 370

Kaiser, N. 1992, ApJ, 388, 272

Kaiser, N. \& Squires, G. 1993, ApJ, 404, 441

Kassiola, A., \& Kovner, I. 1993, ApJ, 417, 450

Kochanek, C. S., \& Blandford, R. D. 1987, ApJ, 321, 676

Kochanek, C. S. 1991, ApJ, 382, 58

Kochanek, C. S. 1993, ApJ, 419, 12

Kochanek, C. S. 1995, ApJ, 445, 559

Kormann, R., Schneider, P., \& Bartelmann, M. 1994, A\&A, 286, 357

Lehár, J., Hewitt, J. N., Roberts, D. H., \& Burke, B. F. 1992, ApJ, 384, 453

Narasimha, D., Subramanian, K., \& Chitre, S. M. 1982, MNRAS, 200, 941

Narayan, R. 1991, ApJ, 378, L5

Pelt, J., Hoff, W., Kayser, R., Refsdal, S., \& Schramm, T. 1994, A\&A, 286, 775

Pelt, J., Kayser, R., Refsdal, S., \& Schramm, T. 1995, A\&A, in press

Porcas, R. W., Booth, R. S., Browne, I. W. A., Walsh, D., \& Wilkinson, P. N. 1981, Nature, 289,758

Press, W. H., Flannery, B. P., Teukolsky, S. A., \& Vetterling, W. T. 1992, Numerical Recipes (Second Edition, Cambridge: Cambridge Univ. Press) 
Press, W. H., Rybicki, G. B., \& Hewitt, J. N. 1992b, ApJ, 385, 404

Press, W. H., Rybicki, G. B., \& Hewitt, J. N. 1992c, ApJ, 385, 416

Refsdal, S. 1964, MNRAS, 128, 295

Refsdal, S. 1966, MNRAS, 132, 101

Rhee, G. 1991, Nature, 350, 211

Rhee, G., Fischer, P., \& Tyson, J. A. 1995, in AIP Conference Proceedings No. 336: Dark Matter, College Park, Maryland, 1994, ed. S. S. Holt \& C. L. Bennett (New York: AIP Press)

Roberts, D. H., Greenfield, P. E., Hewitt, J. N., Burke, B. F., \& Dupree, A. K. 1985, ApJ, 293,356

Schild, R. E. 1990, Lect. Not. Phys., 360, 102

Schild, R. E. \& Thomson, D. J. 1993, in Gravitational Lenses in the Universe: Proceedings of the 31st Liège International Astrophysical Colloquium, June 21-25, 1993, ed. J. Surdej et al.

Schneider, P., Ehlers, J., \& Falco, E. E. 1992, Gravitational Lenses (Heidelberg: Springer)

Schneider, P. \& Seitz, C. 1995, A\&A, 294, 411

Schramm, T. 1994, A\&A, 284, 44

Shapiro I. I. 1964, Phys. Rev. Lett., 13, 789

Stockton, A. 1980, ApJ, 242, L141

Thomson, D. J., \& Schild, R. E. 1993, in Proceedings of the International Conference on Applications of Time Series Analysis in Astronomy and Meteorology, September 6-10, 1993, Padua

Tremaine, S. D. 1995, private communication

Turner, E. L., Ostriker, J. P., Gott, J. R. I. 1984, ApJ, 284, 1

Turner, E. L. 1990, ApJ, 365, L43

Tyson, J. A., Wenk, R. A., \& Valdes, F. 1990, ApJ, 349, 1 
Vanderreist, C., Schneider, J., Herpe, G., Chevreton, M., Moles, M., \& Wlérick, G. 1989, A\&A, 215, 1

Vanderreist, C., Gosset, E., Remy, M., Swings, J.-P. 1992, Lect. Not. Phys., 406, 107

Wallington, S., \& Narayan, R. 1994, ApJ, 403, 517

Walsh, D., Carswell, R. F., \& Weymann, R. J. 1979, Nature, 279, 381

Wambsganss, J., \& Paczynski, B. 1994, AJ, 108, 1156

Young, P., Gunn, J. E., Kristian, J., Oke, J. B., \& Westphal, J. A. 1980, ApJ, 241, 507

—. 1981, ApJ, 244, 736 


\section{Figure Captions}

Fig. 1.- Contours of $\chi^{2}$ for the best-fit SPLS models as a function of the mass power-law exponent $\eta$ and core radius $\theta_{c}$. Although the overall best model has zero core radius, there is a "valley" of low $\Delta \chi^{2}$ extending to $\theta_{c} \sim 110$ mas. The valley is truncated at larger core radii because these models do not sufficiently demagnify the (unseen) third QSO image.

Fig. 2.- Similar to Figure 1, except that contours of $h_{1.5}$ (solid lines) are overlaid on the contours of lowest $\chi^{2}$ (dotted lines) for fixed values of the mass power-law exponent $\eta$ and core radius $\theta_{c}$.

Fig. 3.- Curve showing the lowest $\chi^{2}$ (abscissa) obtainable for SPLS models producing a given $h_{1.5}$ (ordinate). The shallow and then steepening rise to the right of the minimum is caused by our unorthodox penalty assignment for third image flux (\$3.2). As can be seen in Figure 2, the models giving larger $h_{1.5}$ values correspond to increasing core radius, which are chiefly penalized because they allow increasing flux for the (unseen) third QSO image.

Fig. 4.- Contours of model-predicted G1 stellar velocity dispersion $\sigma$ (in $\mathrm{km} \mathrm{s}^{-1}$, solid lines) are overlaid on the contours of lowest $\chi^{2}$ (dotted lines) for fixed values of the mass power-law exponent $\eta$ and core radius $\theta_{c}$. We assume isotropic stellar orbits and a $1^{\prime \prime}$ slit for the measurement.

Fig. 5.- Curves of $\chi^{2}$ versus model-predicted G1 stellar velocity dispersion $\left\langle v_{\text {los }}^{2}\right\rangle^{1 / 2} \equiv \sigma$ for orbit anisotropies $q=-0.2$ (dotted), 0 (solid), and 0.2 (dashed). The three cases represent stellar orbits which are slightly tangential, isotropic, and slightly radial, respectively. 


\section{FIGURE 1}

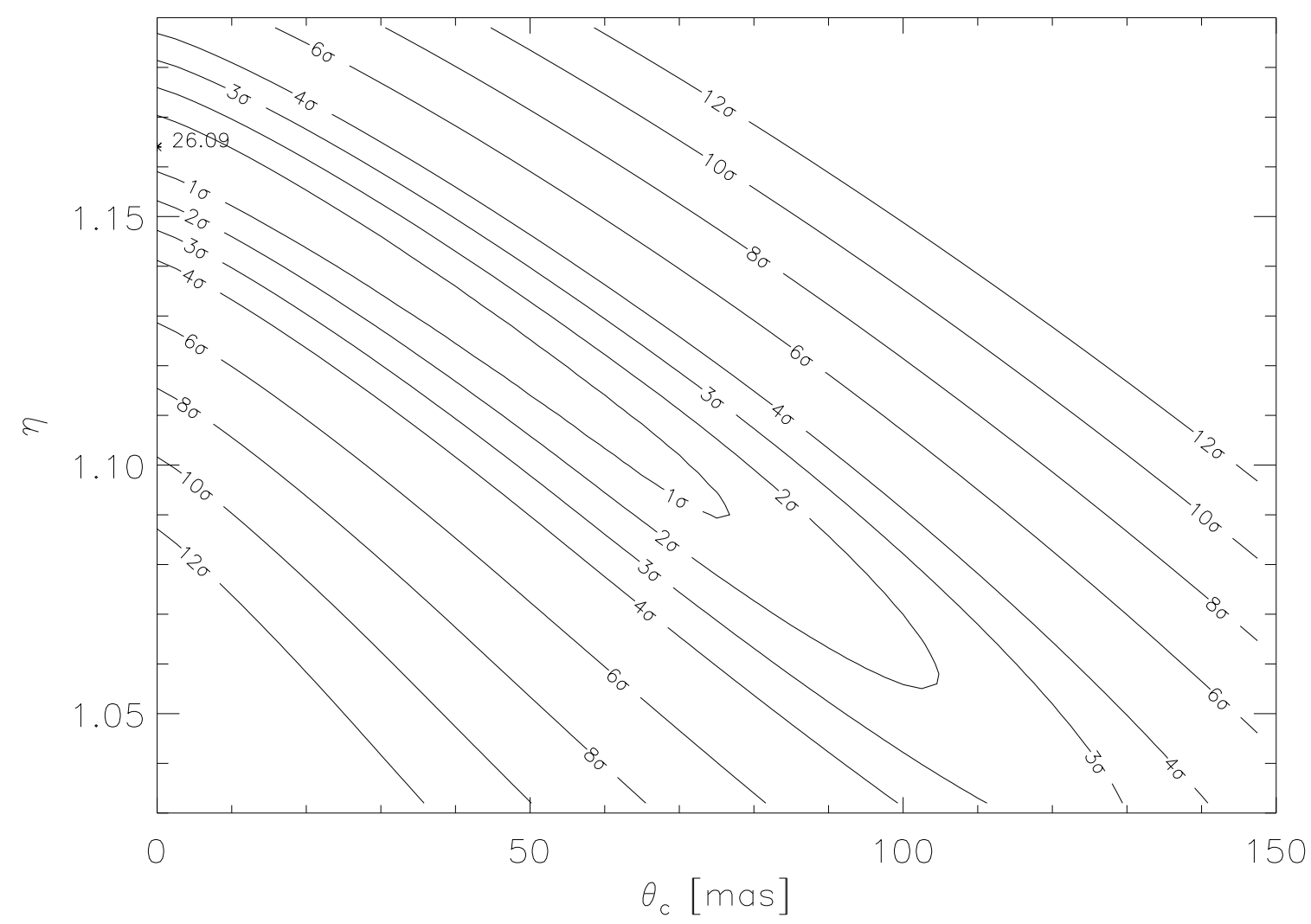




\section{FIGURE 2}

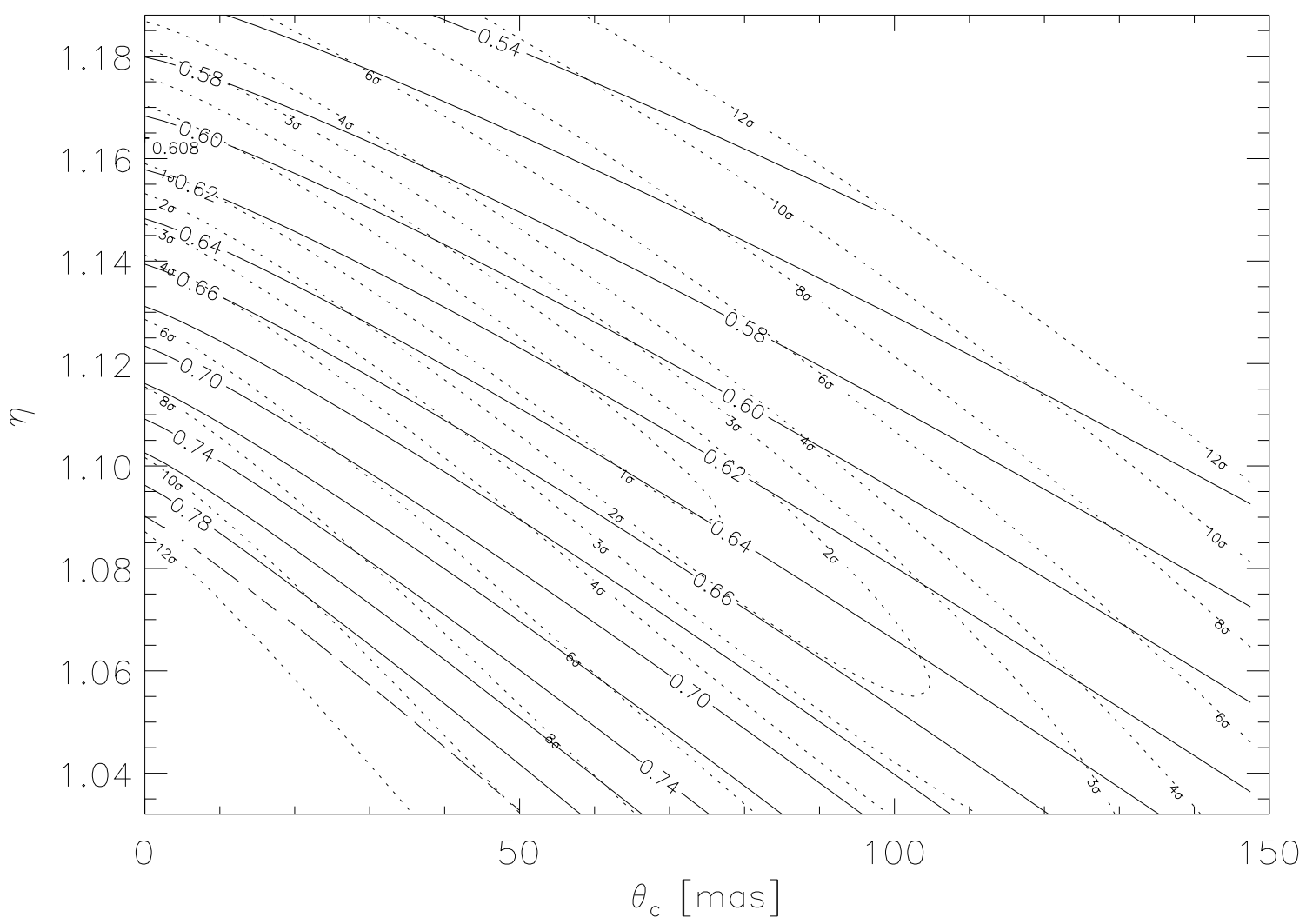




\section{FIGURE 3}

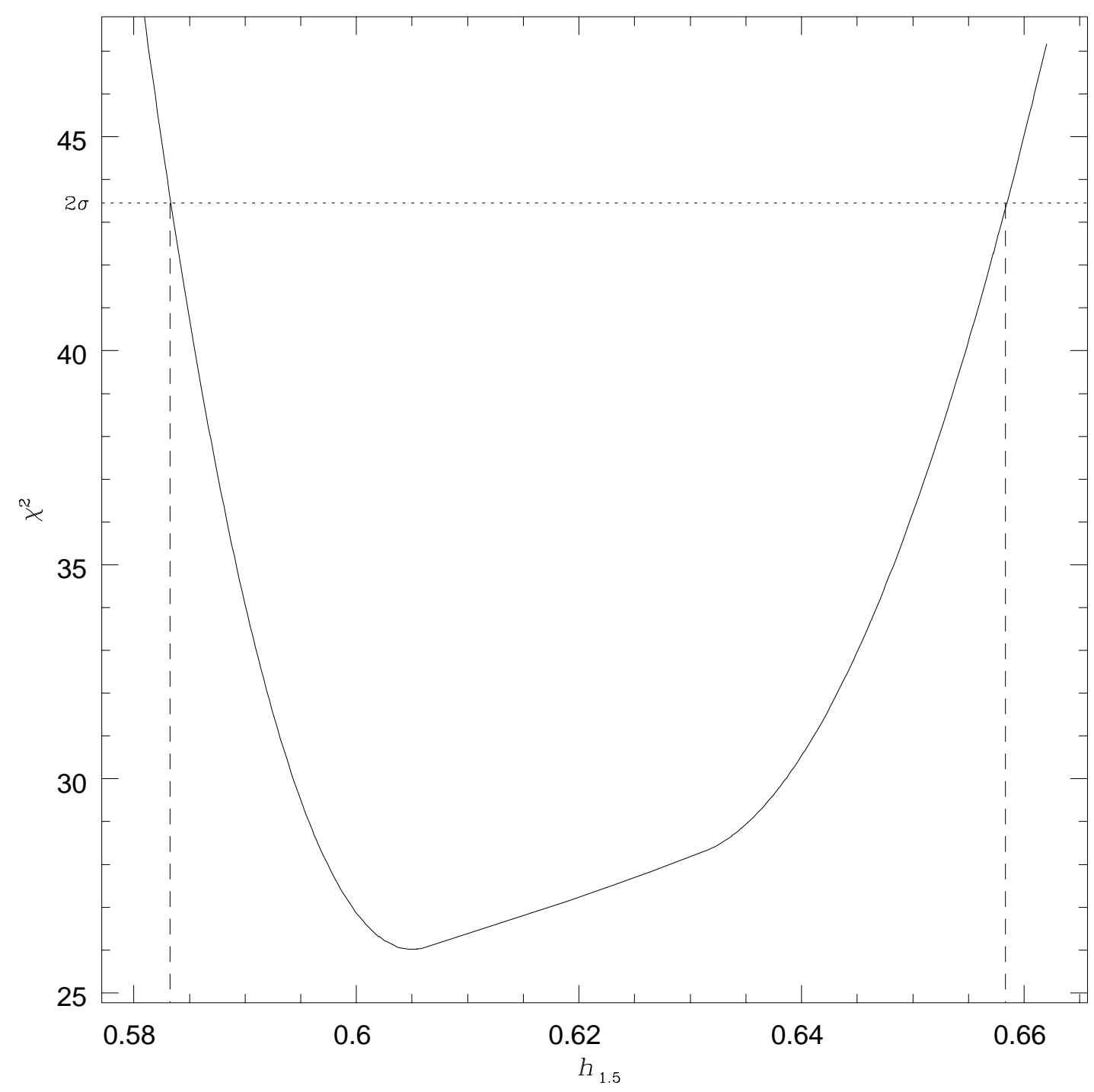




\section{FIGURE 4}

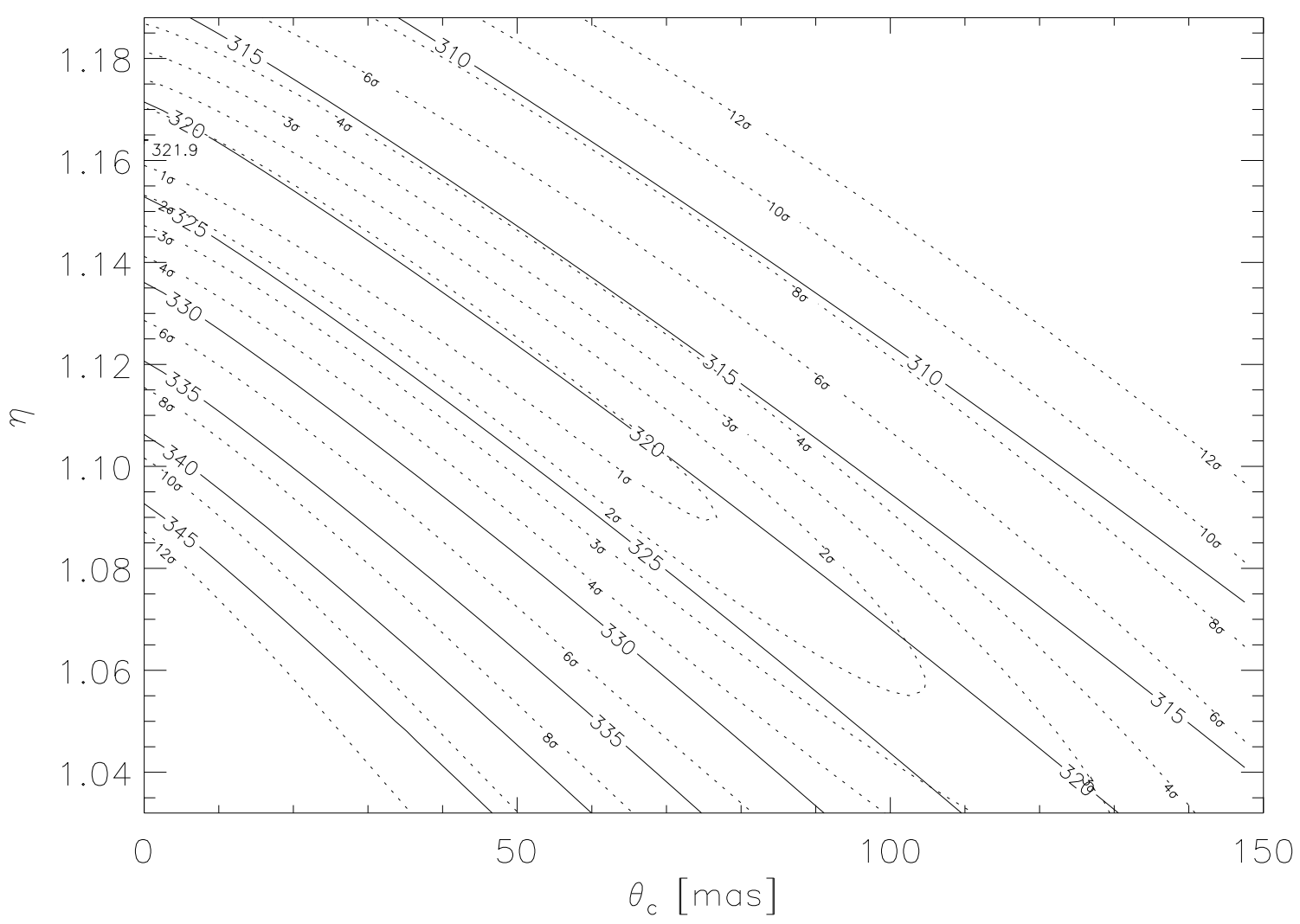




\section{FIGURE 5}

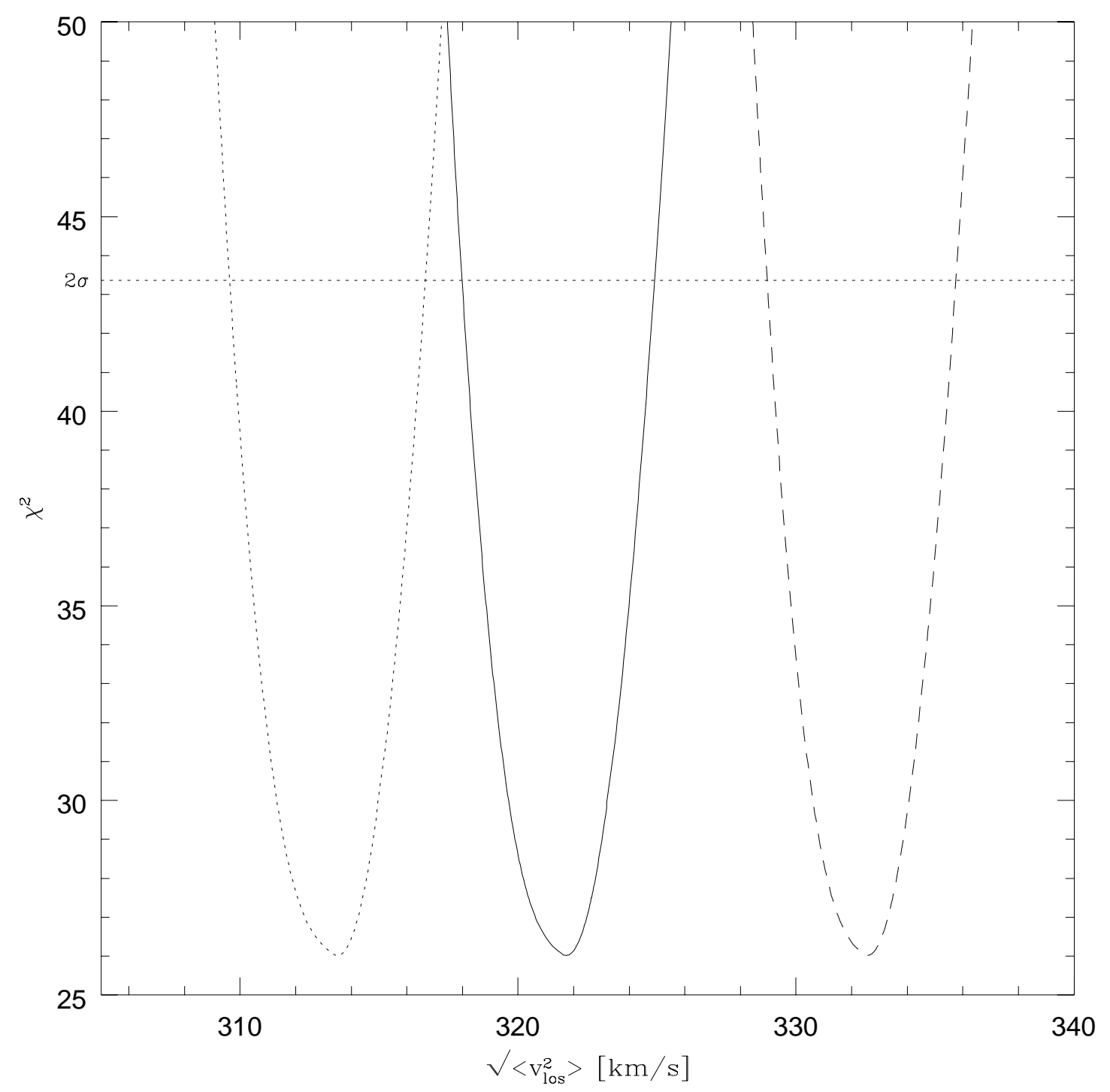

